\title{
Particle acceleration and radiation in flaring complex solar active regions modeled by cellular automata
}

\author{
C. Dauphin ${ }^{1}$, N. Vilmer ${ }^{1}$, and A. Anastasiadis ${ }^{2}$ \\ 1 LESIA, Observatoire de Paris, 92195 Meudon Cedex, France \\ e-mail: cyril.dauphin@obspm. fr \\ 2 National Observatory of Athens, Institute for Space Applications and Remote Sensing, 15236 Penteli, Greece
}

Received 3 March 2006 / Accepted 28 December 2006

\begin{abstract}
Context. We study the acceleration and radiation of electrons and ions interacting with multiple small-scale dissipation regions resulting from the magnetic energy release process.

Aims. We aim to calculate the distribution functions of the kinetic energy of the particles and the X-ray spectra and $\gamma$-ray fluxes produced by the accelerated particles.

Methods. The evolution of the magnetic energy released in an active region is mimicked by a cellular automaton model based on the concept of self-organized criticality. Each burst of magnetic energy release is associated with a reconnecting current sheet (RCS) in which the particles are accelerated by a direct electric field.

Results. We calculate the energy gain of the particles (ions and electrons) for three different magnetic configurations of the RCS after their interactions with a given number of RCS. We finally compare our results with existing observations.

Conclusions. The results of our simulation can reproduce several properties of the observations such as variable electron and ion energy contents and $\gamma$-ray line ratio. Even if very flat X-ray spectra have been reported in a few events, the X-ray spectra produced in this model are too flat when compared to most X-ray observations.
\end{abstract}

Key words. Sun: flares - Sun: X-rays, gamma-rays - acceleration of particles

\section{Introduction}

Solar flares are the result of a sudden and intense release of magnetic energy stored in the solar corona. Magnetic reconnection inside current sheets is believed to be the principal mechanism responsible for the conversion of the magnetic energy to heating and accelerated particles. The importance of particle acceleration in solar flares is strongly supported by many observations of non thermal radiations from energetic particles, which show that a large amount of the magnetic energy is transferred to the accelerated particles (e.g., Saint-Hilaire \& Benz 2005). Different acceleration processes (direct electric field, turbulence, and shocks) have been proposed to explain particle energy fluxes deduced from observations (Miller et al. 1997; or Anastasiadis 2002, for a review).

In this paper, we explore the simplest of these processes: acceleration by the direct electric field that accompanies magnetic reconnection inside a reconnecting current sheet (RCS). In such an approach, acceleration is simply due to the particle motion along the direction of the electric field inside the RCS. However, the particle trajectory and consequently the particle energy gain will depend on the magnetic field configuration in the RCS. The first detailed analysis of particle dynamics within a 2D Harris current sheet configuration was performed by Speiser in 1965. In this pioneering study, two different magnetic field configurations have been considered: with and without a small magnetic field component normal to the reconnecting plane and superposed on the varying magnetic field components in the shield. Following this previous work, particle dynamics in 3D field topologies has been studied by considering a third magnetic field component parallel to the electric field (e.g., Zhu \& Parks 1993; Litvinenko \& Somov 1993; Litvinenko 1996). In such configurations, two different classes of orbits can be defined:

1. The Speiser orbit in which the longitudinal magnetic field is equal to zero. In this case, the Lorentz force due to the presence of the small normal magnetic field component carries the particles out of the RCS.

2. The adiabatic motion in which the longitudinal magnetic field component is large and stabilizes the particle trajectories.

The main conclusion of the studies presented in a series of papers by Litvinenko \& Somov (1993) and Litvinenko (1996) is that the inclusion of a large value of the longitudinal component forces the particles to remain in the current sheet for a longer time, therefore allowing them to gain more energy. In the extreme cases of no longitudinal field or of large longitudinal fields these different studies provide analytical formulae of particle energy gains. More recent analysis of the orbit of charged particles in a 3D field topology of an RCS using dynamical systems methods allow us to re-examine the validity of these analytical formulae, which are mainly valid for electrons with initial small kinetic energies injected at the center of the sheet (Efthymiopoulos et al. 2005). A variation with space of the magnetic field component normal to the reconnection plane has also been considered in more recent studies (e.g., Zharkova \& Gordovskyy 2005a,b; Wood \& Neukirch 2005), which confirm that particle energy gain depends on the injection point in the current sheet and 


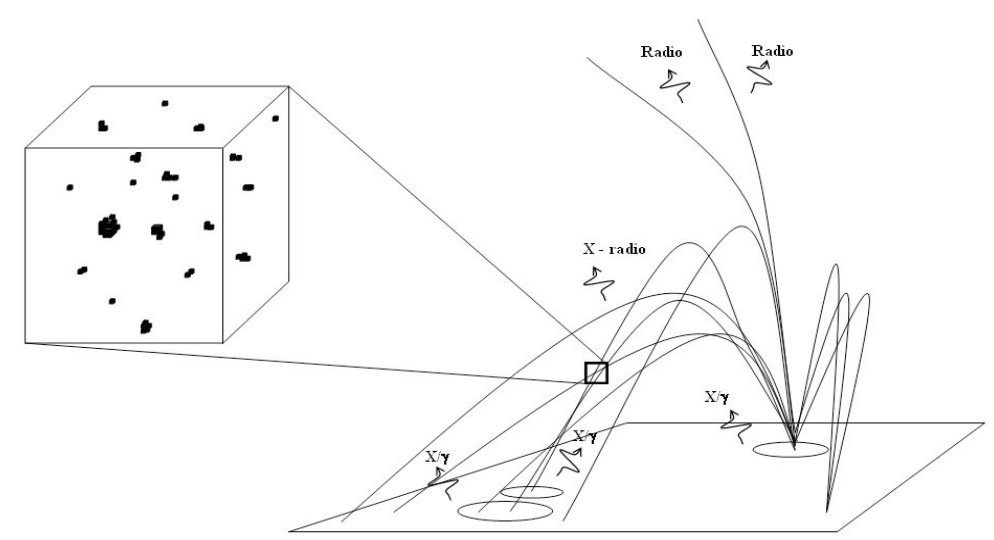

Fig. 1. Sketch of the acceleration model. The location of the main region of magnetic energy release is determined by the topology of the magnetic field and symbolized with a box. The zoom of this box shows the fragmentation of this region in several small regions. This fragmentation is mimicked by a cellular automaton model. Particles are accelerated in these small regions. Outside of the main region of magnetic energy release, particles propagate along the magnetic field line and emit hard X-ray and gamma radiations after their collision with the ambiant plasma.

may even lead to power-law energy spectra for particles escaping from the sheet. Many authors have also performed numerical studies of particle acceleration processes in RCS (e.g., Martens 1988; Martens \& Young 1990; Zharkova \& Gordovskyy 2004; Gontikakis et al. 2006).

In the solar corona, large-scale and long lasting current sheets in which a large number of particles can be accelerated are unlikely to be present. Indeed, recent MHD simulations show that large current sheets are not stable for a long time and are quickly fragmented (e.g., Onofri et al. 2006). This has lead to the development of particle acceleration models considering the presence of multiple current sheets in complex solar active regions (see e.g., Anastasiadis et al. 1997, 2004; Vlahos et al. 2004). This is one of the ways to link acceleration models to energy release processes in a complex active region. Indeed, although the topology of the large-scale magnetic field determines the location of the magnetic energy release regions at the scale of the active region, the complex magnetic environment of an active region as well as the turbulent nature of photospheric motions that externally and continuously drives the system will add new stresses to the existing large-scale topologies and lead to the formation of short-lived, small-scale magnetic discontinuities and current sheets (see e.g., Vlahos et al. 2004; Fragos et al. 2004). This was initially suggested by Parker (1988) and further confirmed by 3D MHD numerical simulations (see, e.g., Nordlund \& Galsgaard 1996). Furthermore, Hughes et al. (2003) have shown that solar flares can be considered as cascades of reconnecting small-scale magnetic loops inside an overall simple large magnetic field topology. These different arguments support the scenario that the magnetic energy release occurs at very small scales when compared to the active region scale and that the current sheets, formation and disappearance should be modeled as a complex and dynamical system (e.g., Vlahos et al. 2004, and references therein).

Several authors have already investigated the problem of particle acceleration by multiple acceleration regions by using either a p-model (Decamp \& Malara 2006), MHD simulations combined with a relativistic test particle code (Dmitruk et al. 2003, 2004; Turkmani et al. 2005; Onofri et al. 2006), or cellular automaton (CA) models. CA models based on the concept of self organized criticality (SOC) can indeed mimic the complex evolution of the magnetic energy released in a solar flare and have successfully reproduced several statistical properties of solar flares such as peak flux or total flux distributions (Vlahos et al. 1995; Georgoulis \& Vlahos 1998).

Previous studies trying to link the complexity of the energy release process to the particle acceleration process have considered that each burst of energy release is due to a reconnecting process. While Anastasiadis \& Vlahos $(1991,1994)$ considered electron acceleration by an ensemble of shock waves generated by multiple energy release processes, Anastasiadis et al. (1997) considered electron acceleration by direct electric fields generated in a CA model. In this paper, we have continued this last approach, but we consider the dependence of the particle energy gain on the RCS magnetic configuration. It follows that electrons, protons, and heavier ions may have different energy gains resulting in different energy spectra. Hard X-ray spectra and gamma ray fluxes produced by the energetic particles will thus depend on the magnetic field configuration of every elementary acceleration site. We shall compare our theoretical hard $\mathrm{X}$-ray spectra and gamma ray ratio to results derived from SMM and RHESSI observations.

A sketch of the model used in this paper is shown in Fig. 1. While the location of the main region of magnetic energy release is determined by the topology of the large-scale magnetic fields, particles are accelerated inside the reconnecting current sheets created at small scales and distributed in the overall largescale structures. The distribution of magnetic energy release inside these RCSs is given by a CA model. Outside of the main region of magnetic energy release, particles propagate freely along the magnetic field lines and are supposed to emit hard X-ray and gamma-ray radiations in the dense regions of the solar atmosphere (thick target approximation).

The paper is organized as follows: Sect. 2 gives the details of the CA model, while we present the details of the acceleration model in Sect. 3. Resulting particle energy spectra are presented in Sect. 4. In Sect. 5, we discuss the hard X-ray spectra and gamma ray fluxes obtained. In the last section, we discuss the different results and the validity of the model.

\section{The CA model for energy release}

In this work, we use a CA model evolving in the SOC state to model the spatial and temporal evolution of these dissipative regions. The basic evolution rules for the $\mathrm{CA}$ model are derived from the CA model of Vlahos et al. (1995) and Georgoulis \& Vlahos (1998) and are described below (see 
detailed description in Vlahos et al. 1995; and Georgoulis \& Vlahos 1998).

1. The loading of the magnetic field:

A scalar magnetic field is associated with each point of a 3D cubic grid that represents a part of a complex active region. The initial configuration of the magnetic field is random and stable. During a flare, the action of the photosphere as a turbulent driver of the magnetic field is simulated by an increase of the field $\delta B$ at a random point of the selected grid. The scalar increment $\delta B$ is given by a power law probability with a slope $-5 / 3$ :

$P(\delta B)=K(\delta B)^{-\frac{5}{3}}$,

where $P(\delta B)$ is the probability of an increment $\delta B$. It can be noted that some observations of power spectra of the solar granulation indeed show power-law distributions of velocity and intensity fluctuations with a slope $-5 / 3$, characteristic of a turbulent atmosphere (see, e.g., Espagnet et al. 1993).

2. Instability criterion:

The evolution of the magnetic field is based on a simple instability criterion that is related to the diffusion of the magnetic field, and thus controlled by its gradient. In this way, the quantity that triggers the instability at a given point $i$ is given by:

$S_{i}=B_{i}-\frac{1}{6} \sum_{j} B_{j}$,

where the index $j$ defines the neighbors of the point $i$. The relaxation of the magnetic field occurs when the instability criterion is satisfied:

$S_{i} \geq B_{\text {cr }}$,

where $B_{\mathrm{cr}}$ is a critical value of the magnetic field. In this model, $B_{\text {cr }}$ is equal to 10 as in Vlahos et al. (1995).

3. Field restructuring:

The evolution of the magnetic field during the relaxation process is given by (details in Vlahos et al. 1995):

$B_{i}(t+1)=B_{i}(t)-\frac{6}{7} B_{\mathrm{cr}}$

$B_{j}(t+1)=B_{j}(t)+\frac{1}{7} B_{\mathrm{cr}}$.

The diffusion of the magnetic field at the point $i$ leads to an increase of the field at the neighbors $j$. If one of the neighbors satisfies the instability criterion, the field at this point is then relaxed according to the same rules. This process might lead to a continuous restructuration of the magnetic field and to the trigger of an avalanche process. Isliker et al. (1998) showed how the equations used to describe the basic rules of the CA model can be linked to the induction equation of the magnetic field.

4. Energy release:

Magnetic energy $\varepsilon_{i}(t)$ is released at each point that satisfies the instability criterion according to:

$\varepsilon_{\mathrm{Ri}}(t)=\left(B_{i}(t)-\frac{6}{7} B_{\mathrm{cr}}\right)^{2}=B_{\mathrm{o}}^{2}$.

The time evolution of the magnetic field determined by the restructuring and the avalanche process produces energy release time series as illustrated in Fig. 2. As shown in Fig. 3, the distribution of the magnetic field energy is close to a power law with a slope of -1.6 (see Vlahos et al. 1995, for details).

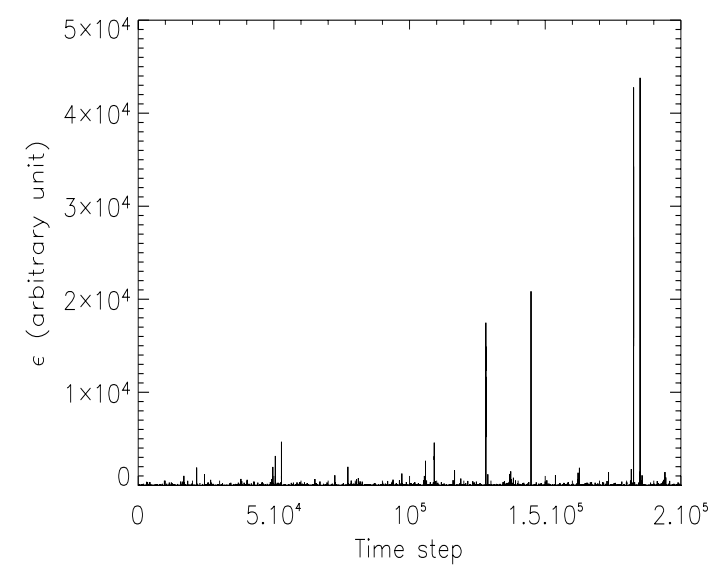

Fig. 2. Energy release time series given by the CA model.

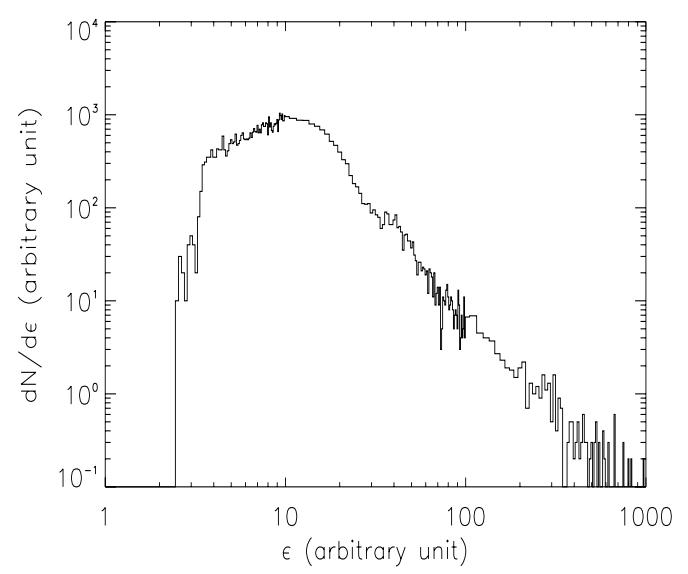

Fig. 3. Distribution of the magnetic energy release given by the CA model.

\section{Acceleration model}

Each burst of magnetic energy release in the CA model is assumed to represent an elemental magnetic reconnection process. The energy release time series of Fig. 2 thus generates a time series of reconnecting current sheets (RCS). To predict the production of energetic particles from these RCS, it is now necessary to study the link between energy release and acceleration processes. A first step had been performed for electrons by Anastasiadis et al. (1997) and Anastasiadis et al. (2004).

\subsection{Determination of the electric field in the reconnecting current sheet}

Figure 4 shows the geometry of one elemental RCS. The first step of the present model is to relate the electric field seen by the particles in each RCS to the magnetic energy $\left(\propto B_{0}^{2}\right)$ released in the CA model. This is done by equating the magnetic energy flux into the sheet to the energy gained by the accelerated particles per unit time. The magnetic energy flux into the sheet can be expressed as a function of the inflow speed $\left(v_{\text {in }}\right)$ :

$P=\frac{1}{\pi} v_{\text {in }} B_{0}^{2} b l$,

where $l$ and $b$ are, respectively, the length and width of the RCS and $B_{0}$ is the field outside the RCS deduced from Eq. (6).

When considered individually, particles have different trajectories in a given RCS depending on their initial velocity and 


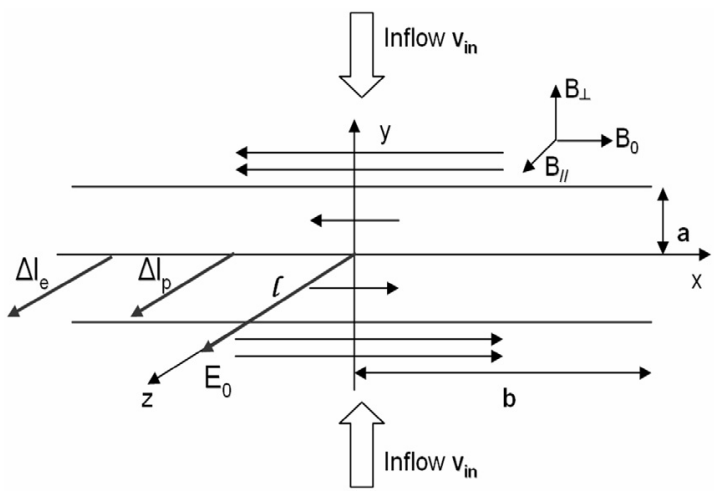

Fig. 4. Geometry of one elemental reconnecting current sheet.

on the injection point in the current sheet, thus implying different acceleration lengths $\Delta l$. This may create power-law energy distributions for particles escaping from a single current sheet (see, e.g., Zharkova \& Gordovskyy 2005a; Wood \& Neukirch 2005). In this work no simulation of particle trajectories is performed and we consider that all particles injected in one RCS follow a mean trajectory and thus are accelerated on a mean acceleration length. We thus define $\left\langle\Delta l_{\mathrm{e}}\right\rangle_{n_{\mathrm{e}}}$ and $\left\langle\Delta l_{\mathrm{p}}\right\rangle_{n_{\mathrm{p}}}$ as, respectively, the average acceleration lengths of electrons and protons incoming in one RCS. The energy gained per unit time by the accelerated particles can then be written as:

$P=\dot{N}_{\mathrm{e}} e E_{0}\left\langle\Delta l_{\mathrm{e}}\right\rangle_{n_{\mathrm{e}}}+\dot{N}_{\mathrm{p}} e E_{0}\left\langle\Delta l_{\mathrm{p}}\right\rangle_{n_{\mathrm{p}}}$

with $\dot{N}_{\mathrm{e}, \mathrm{p}}=4 l b v_{\mathrm{in}} n_{\mathrm{e}, \mathrm{p}}$ implying:

$P=4 l b v_{\text {in }} n_{\mathrm{e}} e E_{0}\left\langle\Delta l_{\mathrm{e}}\right\rangle_{n_{\mathrm{e}}}+4 l b v_{\text {in }} n_{\mathrm{p}} e E_{0}\left\langle\Delta l_{\mathrm{p}}\right\rangle_{n_{\mathrm{p}}}$,

where $\dot{N}_{\mathrm{e}, \mathrm{p}}$ is the number of electrons and protons injected in the sheet per unit time, $n_{\mathrm{e}}$ and $n_{\mathrm{p}}$ are respectively the density of electrons and protons outside the sheet, and $E_{0}$ is the constant electric field inside the sheet. We neglect in this expression the flux and energy gain of ions heavier than protons since they are less abundant than protons. Equating (7) and (9) leads to the following expression for the electric field seen by the particles in the sheet:

$E_{0}=\frac{B_{0}^{2}}{4 \pi e\left(\left\langle\Delta l_{\mathrm{e}}\right\rangle_{n_{\mathrm{e}}} n_{\mathrm{e}}+\left\langle\Delta l_{\mathrm{p}}\right\rangle_{n_{\mathrm{p}}} n_{\mathrm{p}}\right)}$.

Note that in this work, there is no condition on the inflow speed value that triggers the reconnection. The electric field depends only on the magnetic energy released, derived from the $\mathrm{CA}$ model, and on the mean acceleration length of electrons and protons.

\subsection{Particle energy gain}

The energy gain of one particle in a RCS with electric field $E_{0}$ is given by:

$\varepsilon= \pm e E_{0} \Delta l$.

The plus or minus sign corresponds to the fact that the particle velocity can be parallel or anti-parallel with respect to the direction of the electric field. $\Delta l$ is the acceleration length of one individual particle and can be expressed as a function of the average acceleration length $\left\langle\Delta l_{\mathrm{e}}\right\rangle_{n_{\mathrm{e}}}$ and $\left\langle\Delta l_{\mathrm{p}}\right\rangle_{n_{\mathrm{p}}}$ in the way described below.
In the present model, we make the simple assumption that individual trajectories are similar to average trajectories. This implies an ergodic hypothesis in which the average on the total number of particles at a given time is similar to the average acceleration lengths on many particle trajectories in a single current sheet. However, this does not take into account the efficiency of particle acceleration defined in the following by $\alpha$. In the following, the acceleration efficiency $\alpha$ at each interaction is a random number selected between zero and one. Thus, the efficient acceleration length for one particle is given by:

$\Delta l=\alpha\langle\Delta l\rangle$.

In the case when all the released energy would go to acceleration, $\alpha$ would be equal to one and, on the contrary, would be zero if the released magnetic energy released goes in totality into, e.g., heating. This choice for $\alpha$ shall be discussed in the last part of the paper. Let us now introduce $\beta$ as:

$\beta=\frac{\left\langle\Delta l_{\mathrm{p}}\right\rangle_{n_{\mathrm{p}}}}{\left\langle\Delta l_{\mathrm{e}}\right\rangle_{n_{\mathrm{e}}}}$,

The energy gain for electrons and protons can now be expressed as:

$\varepsilon= \pm e E_{0} \alpha\left\langle\Delta l_{\mathrm{e}}\right\rangle_{n_{\mathrm{e}}}$,

where $E_{0}$ is given by Eq. (10). This implies that:

$\varepsilon_{\mathrm{e}}= \pm \alpha \frac{B_{0}^{2}}{4 \pi\left(n_{\mathrm{e}}+\beta n_{\mathrm{p}}\right)}$

$\varepsilon_{\mathrm{p}}= \pm \alpha \beta \frac{B_{0}^{2}}{4 \pi\left(n_{\mathrm{e}}+\beta n_{\mathrm{p}}\right)}$.

For ions with $Z$ as the atomic number of the element, the energy gain is:

$\varepsilon_{\mathrm{i}}= \pm \alpha Z \beta_{\mathrm{i}} \frac{B_{0}^{2}}{4 \pi\left(n_{\mathrm{e}}+\beta n_{\mathrm{p}}\right)}$,

where $Z$ is the atomic number of the element and:

$\beta_{\mathrm{i}}=\frac{\left\langle\Delta l_{\mathrm{i}}\right\rangle_{n_{\mathrm{i}}}}{\left\langle\Delta l_{\mathrm{e}}\right\rangle_{n_{\mathrm{e}}}}$

$\left\langle\Delta l_{\mathrm{i}}\right\rangle_{n_{\mathrm{i}}}$ is the average acceleration length of an ion with $Z$ as an atomic number. Equations (15)-(17) link the energy release process characterized by the magnetic energy $B_{0}^{2}$ to the acceleration process. These formulae represent a general manner to link models that mimic the evolution of the magnetic field in an active region, such as $\mathrm{MHD}, \mathrm{CA}$, or XCA (i.e., extended CA, see Isliker et al. 2001) models, to particle acceleration models.

\subsection{Particle acceleration lengths in an RCS}

The parameter $\beta$ (ratio of the average acceleration lengths) is estimated here by investigating the acceleration process by a direct electric field in a single RCS. We use the simple configuration of a RCS given in, e.g., Litvinenko (1996).

$B_{\mathrm{RCS}}=\left(-\frac{y}{a} B_{0}, B_{\perp}, B_{/ /}\right), E_{\mathrm{RCS}}=\left(0,0, E_{0}\right)$,

where $a$ is the thickness of the RCS (see Fig. 4). The ratio of the acceleration lengths (Eqs. (13) and (18)) is estimated for three different assumptions of the value of the longitudinal magnetic field $B_{/ /}$. 


\subsubsection{RCS with no longitudinal magnetic field}

In the case of an RCS with no longitudinal magnetic fields, the particle energy gain is the same for all species. With the hypothesis that initial velocities are negligible compared to final velocities, the energy gain is given by (Speiser 1965):

$\varepsilon=2 m c^{2}\left(\frac{E_{0}}{B_{\perp}}\right)^{2}$.

From Eq. (11), the maximum energy gain, with an acceleration efficiency of one, is given by $\varepsilon=e E_{0} \Delta l=e E_{0}\langle\Delta l\rangle$ if we assume that all particles follow the Speiser trajectories. Equating the two previous equations for both electrons and protons allows to find the ratio $\beta$ :

$\beta=\frac{m_{\mathrm{p}}}{m_{\mathrm{e}}}$.

Similarly, for ions with atomic number $Z$, we have:

$\beta_{\mathrm{i}}=\frac{m_{\mathrm{i}}}{Z m_{\mathrm{e}}}$

Inserting expressions (21) and (22) into Eqs. (15)-(17) shows that electrons gain less energy than ions. Indeed, electrons and ions leave the RCS with the same speed (Speiser 1965). Most of the energy provided by the RCS to the accelerated particles goes to ions. Finally, each kind of ions gain the same energy per nucleon as the energy gain is proportional to the mass number $\mathrm{A}$.

\subsubsection{RCS with a large longitudinal magnetic field}

In this case, we assume that the longitudinal magnetic field in all the RCS is large enough so that all particle species are fully magnetized and follow magnetic field lines through the whole RCS (Litvinenko \& Somov 1993; Litvinenko 1996). From the movement of the particle in one RCS, we may deduce:

$\Delta l_{\mathrm{e}, \mathrm{p}, \mathrm{i}}=\frac{B_{/ /}}{B_{\perp}} a=\langle\Delta l\rangle_{\mathrm{e}, \mathrm{p}, \mathrm{i}}$

so that

$\beta=\beta_{i}=1$.

Equations (15)-(17) then lead to the following expression for the particle energy gain:

$\varepsilon_{\mathrm{e}}=\varepsilon_{\mathrm{p}}= \pm \alpha \frac{B_{0}^{2}}{4 \pi\left(n_{\mathrm{e}}+\beta n_{\mathrm{p}}\right)}$.

This equation has already been used in Anastasiadis et al. (2004) for electron acceleration and neglecting protons in the RCS. We show here that in this paper, the calculation was computed in the approximation of a large longitudinal magnetic field in which electrons are always magnetized. For ions heavier than protons, we find:

$\varepsilon_{\mathrm{i}}= \pm \alpha Z \frac{B_{0}^{2}}{4 \pi\left(n_{\mathrm{e}}+n_{\mathrm{p}}\right)}$.

In this case, electrons and protons gain the same energy in each RCS in which they are fully magnetized. In comparison with the previous case for a given value of $E_{0}$, electrons gain more energy and protons gain less energy because the electromagnetic power is equally divided here between electrons and protons. For other ions, the energy gain per nucleon is proportional to the ratio $Z / A$.

\subsubsection{RCS with an intermediate longitudinal magnetic field}

In the previous subsection we made the hypothesis that electrons and ions are fully magnetized in all the RCS. We consider here the case where the longitudinal magnetic field in a RCS always magnetizes electrons but not protons nor ions. Indeed, as expected from Eq. (27), protons and heavier ions are more difficult to magnetize than electrons since the critical longitudinal magnetic field required to magnetize particles is larger for protons and heavy ions than for electrons (e.g., Litvinenko \& Somov 1993):

$B_{/ / \mathrm{ce}, \mathrm{p}}=\sqrt{\frac{m c^{2} E_{0} B_{0}}{e a B_{\perp}}}$.

In the present case, each particle will interact with a number of RCS that may have different longitudinal magnetic fields. For the total number of RCS encountered by a particle, we then define a probability $p\left(B_{/ / \mathrm{c}}\right)$ that the longitudinal magnetic field can magnetize the particle. Let us introduce $\gamma$ as the ratio between the probabilities that a given RCS magnetizes electrons or protons:

$\gamma=\sqrt{\frac{m_{\mathrm{e}}}{m_{\mathrm{p}}}}$

Note that if all the RCS magnetize electrons, we shall have $p_{/ /}\left(B_{\mathrm{ce}}\right)=1$ for all RCS. The energy gained by electrons after encountering N RCS is given by:

$G_{\mathrm{e}}=\sum_{j=1}^{N} \varepsilon_{\mathrm{e}}(j) p\left(B_{/ / \mathrm{ce}}\right)_{j}=N\left\langle\varepsilon_{\mathrm{e}}\right\rangle_{N}$.

We define $\varepsilon_{\mathrm{e}}(j)$ as the energy gain in one RCS and $\left\langle\varepsilon_{\mathrm{e}}\right\rangle_{N}$ as the average over the number of RCS. We suppose that $\varepsilon_{\mathrm{e}}(j)$ is given by Eq. (25) when the electrons are magnetized. Similarly, for protons, the energy gain after encountering N RCS is given by:

$G_{\mathrm{p}}=\sum_{j=1}^{N} \varepsilon_{\mathrm{p}}(j) p\left(B_{/ / \mathrm{cp}}\right)_{j}=N\left\langle\varepsilon_{\mathrm{p}}\right\rangle_{N}$

We suppose that $\varepsilon_{\mathrm{p}}(j)$ is given by Eq. (25) when the protons are magnetized and assume that the energy gain is negligible when protons are not magnetized. With Eq. (28), this gives:

$G_{\mathrm{p}}=\sum_{j=1}^{N} \gamma \varepsilon_{\mathrm{p}}(j) p\left(B_{/ / \mathrm{ce}}\right)$

As shown in the previous section (Eq. (25)), protons and electrons gain the same energy when particles are magnetized and:

$$
\begin{aligned}
& G_{\mathrm{p}}=\gamma \sum_{j=1}^{N} \varepsilon_{\mathrm{e}}(j) p\left(B_{/ / \mathrm{ce}}\right) \\
& G_{\mathrm{p}}=N \gamma\left\langle\varepsilon_{\mathrm{e}}\right\rangle_{N} .
\end{aligned}
$$

The average proton and electron energy gains are then related by:

$\left\langle\varepsilon_{\mathrm{p}}\right\rangle_{N}=\gamma\left\langle\varepsilon_{\mathrm{e}}\right\rangle_{N}$.

This equation gives the ratio of the average proton to electron energy gains, which is easily related to the ratio $\beta$ of the acceleration lengths of electrons and protons:

$\gamma=\frac{\left\langle\varepsilon_{\mathrm{p}}\right\rangle_{N}}{\left\langle\varepsilon_{\mathrm{e}}\right\rangle_{N}}=\frac{\left\langle\Delta l_{\mathrm{p}}\right\rangle_{n_{\mathrm{p}}}}{\left\langle\Delta l_{\mathrm{e}}\right\rangle_{n_{\mathrm{e}}}}=\beta$. 
Based on the same assumptions, the energy gained by ions (charge number $Z$ ) after encountering N RCS will be given by:

$G_{\mathrm{i}}=N Z \gamma_{\mathrm{i}}\left\langle\varepsilon_{\mathrm{e}}\right\rangle_{N}$

with:

$\gamma_{\mathrm{i}}=\sqrt{\frac{Z m_{\mathrm{e}}}{m_{\mathrm{i}}}}=\beta_{\mathrm{i}}$

Finally, inserting Eqs. (35) and (37) in Eqs. (15)-(17) gives the following expressions for electron, proton, and heavier ion energy gains:

$\varepsilon_{\mathrm{e}}= \pm \alpha \frac{B_{0}^{2}}{4 \pi\left(n_{\mathrm{e}}+\gamma n_{\mathrm{p}}\right)}$

$\varepsilon_{\mathrm{p}}= \pm \alpha \gamma \frac{B_{0}^{2}}{4 \pi\left(n_{\mathrm{e}}+\gamma n_{\mathrm{p}}\right)}$

$\varepsilon_{\mathrm{i}}= \pm \alpha Z \gamma_{\mathrm{i}} \frac{B_{0}^{2}}{4 \pi\left(n_{\mathrm{e}}+\gamma n_{\mathrm{p}}\right)}$.

Compared to the previous case, electrons gain more energy than protons and heavier ions because electrons are always fully magnetized. Ions have an energy gain per nucleon that is proportional to $(Z / A)^{3 / 2}$.

A general comment can be finally made on the principle of the present acceleration model. In all cases, each particle performs a free flight between electric fields of variable strengths, randomly selected from time series with a distribution close to a power law. At each interaction, the particle can randomly gain or lose energy. The evolution of the particle thus follows a Levy flight. Even if the considerations based on the longitudinal magnetic field strength imply different energy gains for electrons, protons, and heavier ions, the general shape of the particle distributions will result from Levy flights and will thus strongly depend on the slope of the electric field distribution.

\section{Final particle energy distributions}

We calculate final particle energy distributions after interactions with a given number $N_{\max }$ of reconnecting current sheets. We consider the three configurations that are detailed in the previous section for each species (i.e., electrons, protons, and heavier ions). Particles are injected in the acceleration volume with an initial Maxwellian distribution with a temperature of $10^{6} \mathrm{~K}$ and with initial velocities in the range $0<V<8 V_{\text {th }}$, where $V_{\text {th }}$ is the thermal velocity. The particle energy distribution for electrons and ions is calculated with a density of $n=10^{10} \mathrm{~cm}^{-3}$.

For each particle, $N_{\max }$ elements in the energy release time series are randomly selected. For each element in the time series, a value of $B_{0}$ is deduced according to Eq. (6) and a random value of $\alpha$ is chosen. Then, depending on the chosen configuration of the RCS, the particle energy gain is computed according to previous expressions. Note that the number $N_{\max }$ of interactions can be an indication of the trapping time of the particles inside the complex active region.

Another free parameter in the present calculations is the physical scaling of the minimum value of the energy release time series considered in the model. This determines the magnitude of the particle energy gain for a given configuration of the RCS since as shown in the previous sections, the particle energy gain depends only on the magnetic energy released and on the density. Equation (10) furthermore shows that the scaling of the magnetic energy released is in fact linked to the normalization of the electric field value in the RCS. The electric field in Eq. (10) computed with the following parameters: $n_{\mathrm{e}}=n_{\mathrm{p}}=n / 2$ and $\left\langle\Delta l_{\mathrm{e}}\right\rangle=\left\langle\Delta l_{\mathrm{p}}\right\rangle=\Delta l=10^{3} \mathrm{~cm}$ is thus $5.5 \times 10^{-4} \mathrm{~V} \mathrm{~cm}^{-1}$. In the following, the scaling will be determined in the case where all RCS magnetize all particle species (i.e., $\left.B_{/ /} \gg B_{\operatorname{mag}(\mathrm{e}, \mathrm{p}, \mathrm{i})}\right)$ (Eq. (25)). The minimum value of the electric field $E_{\min }$ thus computed is then normalized to the Dreicer electric field, and all the RCS that fully magnetize the particles thus have a super Dreicer electric field. The same normalization is kept for the energy release time series when computing particle energy gains for other configurations of the RCS. Three cases are considered for the normalization of the minimum electric field to the Dreicer field: 10, 100 and, 1000. Equation (10) shows that these normalizations correspond to minimum values of the magnetic field $B_{0}$ around $3 \mathrm{G}, 10 \mathrm{G}$ and, $30 \mathrm{G}$, respectively. The maximum values of the magnetic field $B_{0}$ that can be obtained from the magnetic energy release time series are around 100 times the previous values. The final energy distributions of particles presented below are computed for $10^{6}$ particles. This large number of particles ensures that the results are stable and independent of the random number generator.

\subsection{Final electron and proton kinetic energy distributions}

Figures 5 and 6 present final energy distributions for resp. electrons and protons in the different configurations of RCS, for two different values of $N_{\max }$ and for three normalizations of $E_{\min }$ with respect to the Dreicer field $E_{\mathrm{D}}$. The total kinetic energy distribution represented with a dotted line simply represents the sum of the three other distributions. This last case would represent a situation in which RCS with the three different configurations are present in the active region and in which particles will interact with an equal number of RCS in the three different configurations of the magnetic field. Several results can be drawn from these figures:

1. Evolution of the particle energy distributions with the maximum number of interactions $N_{\max }$.

Comparing Figs. 5a, b, c to Figs. 5d, e, f shows that as expected the maximum final energy of the particles increases with the maximum number of interactions. The low energy part of the particle energy distribution calculated for 100 interactions is closer to a power law than the particle energy distribution calculated for 1000 interactions. Indeed in the case of one interaction, the particle energy distribution would be a power law with a spectral index given by the slope of the energy released shown in Fig. 3. With the increase in the number of interactions, each particle has a larger and equal probability of being accelerated by every value of the energy release time series. This results in a spectrum that is close to an inverse exponential function $\mathrm{e}^{-E_{\mathrm{c}}}$ since formally, the particle trajectory is equivalent to a Levy walk.

2. Evolution of the particle energy distributions with the scaling of the minimum electric field value.

As expected, an increase of the scaling of the minimum electric field encountered leads to an increase of the maximum energy gain. The conservation of the particle number also implies a flattening of the energy distribution at low energies far from the high-energy cut-offs.

3. Evolution of the electron energy distributions with the configuration of the RCS. 

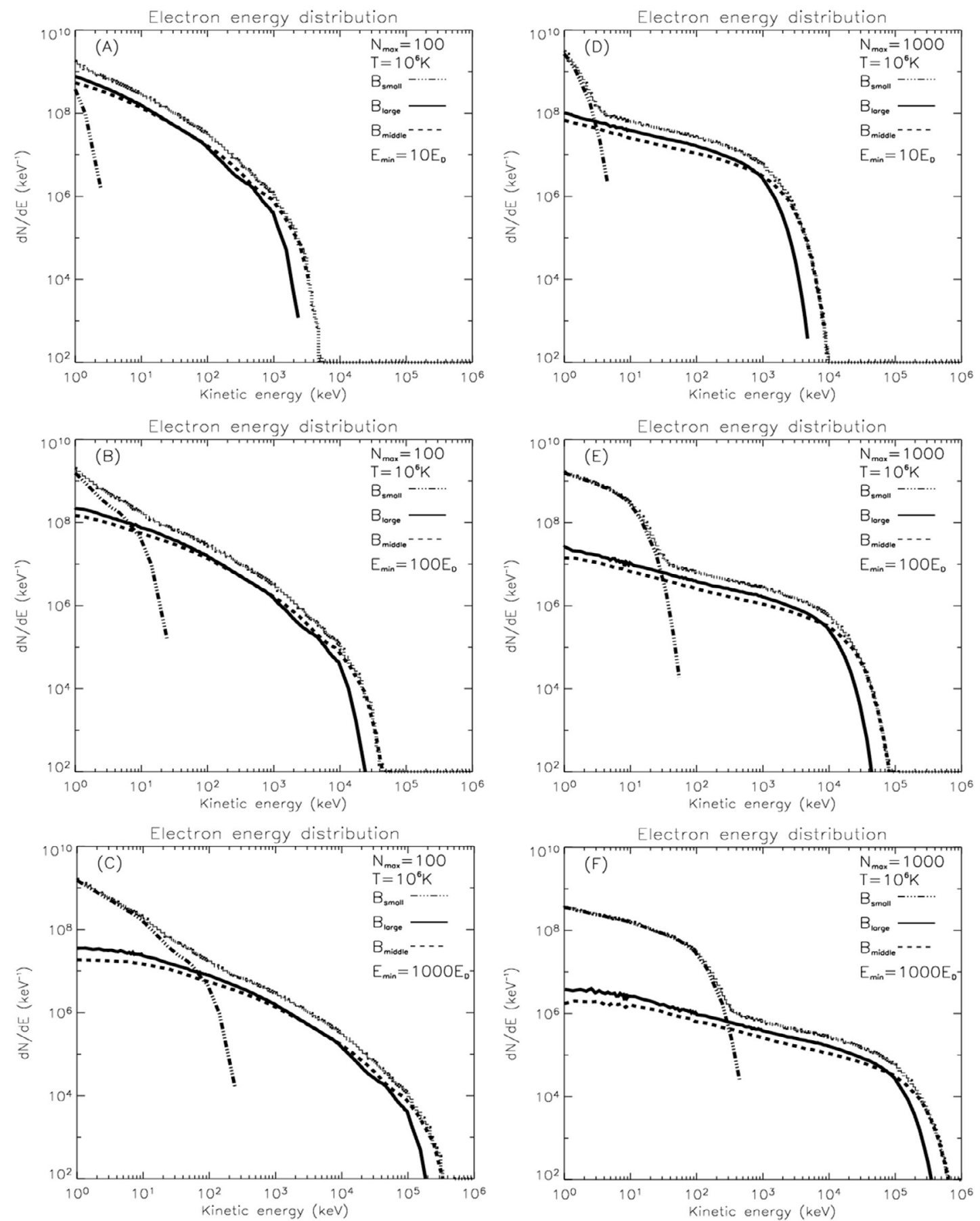

Fig. 5. Panel of the electron energy distribution obtained for the three different electric field normalizations $E_{\min }=10 E_{\mathrm{D}}, E_{\min }=100 E_{\mathrm{D}}$, and $E_{\min }=1000 E_{\mathrm{D}}$ and for the two different maximum numbers of interactions $N_{\max }=100$ and $N_{\max }=1000$. In each panel, the dashed-dotted line corresponds to the distribution in the case $B_{\text {small }}$, the dashed line corresponds to the case $B_{\text {middle }}$, and the black line corresponds to the case $B_{\text {large }}$. The dotted line corresponds to the sum of these three distributions. The bumps at low energies are due to the superposition of the different distributions.

As expected from the discussion in Sects. 3.3.1 and 3.3.2 based on individual electrons, RCS with small values of $B_{/ /}$ are less efficient to accelerate electrons than RCS with large value of $B_{/ /}$that fully magnetize electrons. We recover in these simulations some of the results of Litvinenko (1996, 2000), who showed that electrons can be accelerated in the super Dreicer regime to energies as high as $10 \mathrm{MeV}$ in RCS with strong values of $B_{/ /}$. The artificial break in the total electron spectra close to the high-energy cut-off of the electron distribution is due to the conservation of the electron number in the case of the computation with small longitudinal fields.

4. Evolution of the relative electron and proton energy distributions with the configuration of the RCS.

The results deduced from the final particle energy distributions confirm what is expected from the study of the energy gains in the different configurations. RCS with small values of longitudinal magnetic field strengths provide large energy 

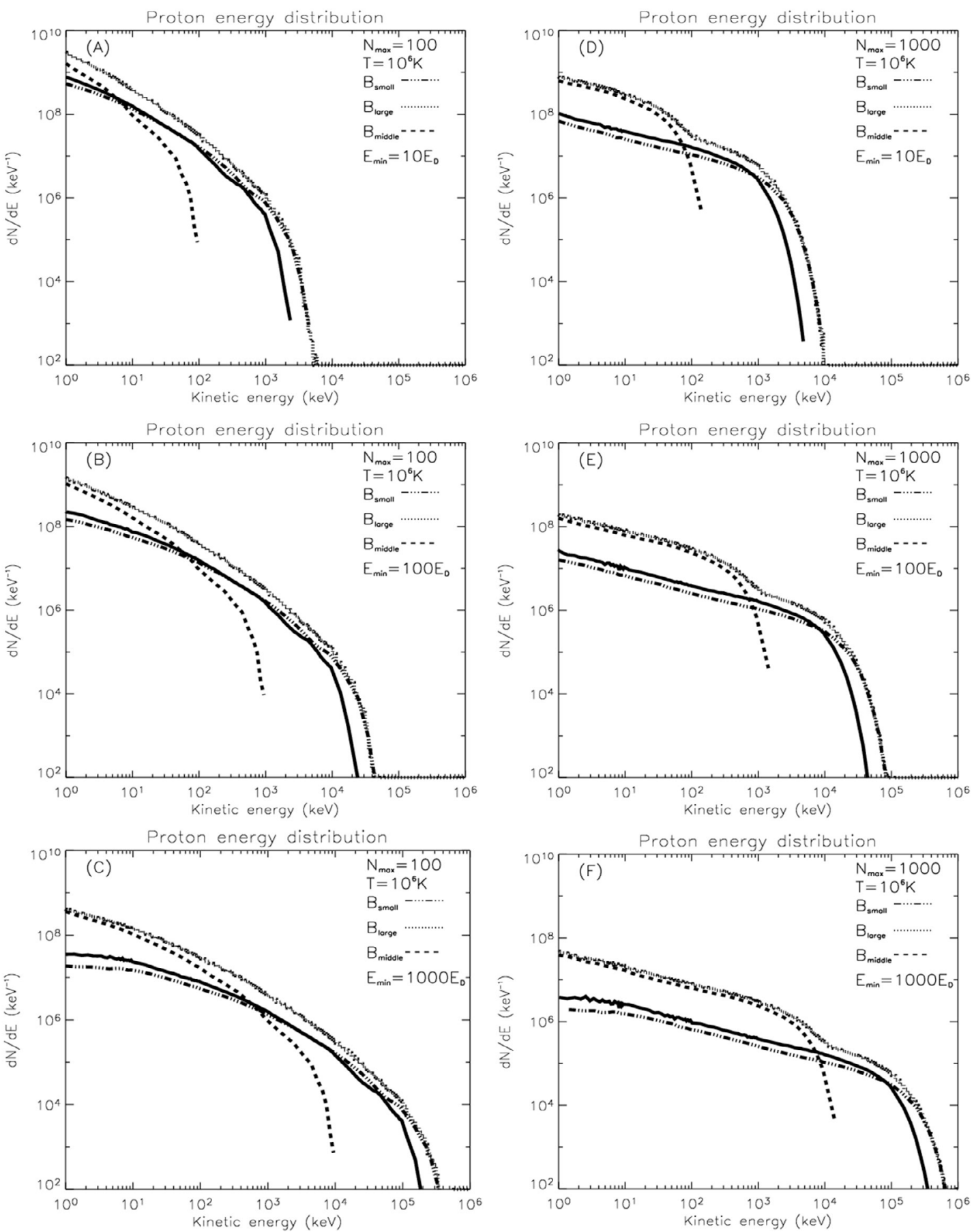

Fig. 6. Same as Fig. 4, but for the case of protons.

gains for protons (Speiser 1965). Electron and proton energy distributions are the same in the case when RCS have longitudinal magnetic fields large enough to magnetize both electrons and protons. In the case with intermediate longitudinal magnetic fields, the final electron energy gain is larger than the final proton energy gain since, contrary to protons, electrons are then assumed to be always magnetized. Finally, the conservation of the total proton numbers for each RCS configuration also creates an artificial break in the total proton energy distributions close to the high-energy cut-off of the proton distribution computed with intermediate longitudinal fields. Finally, the comparison of the final electron and proton energy distributions illustrates the fact that electrons or ions are preferentially accelerated depending on the relative magnitudes of the various components of the magnetic field.

5. Sum of electron and proton distributions in the different configurations

The comparison of the sum of the three energy distributions for electrons and protons (dotted lines in Figs. 5 and 6) shows that the final energies reached by both species are similar. This is related to the choice of the acceleration efficiency $\alpha$ for each interaction in Eq. (12) that has a similar distribution for electrons and protons. The main difference in the 

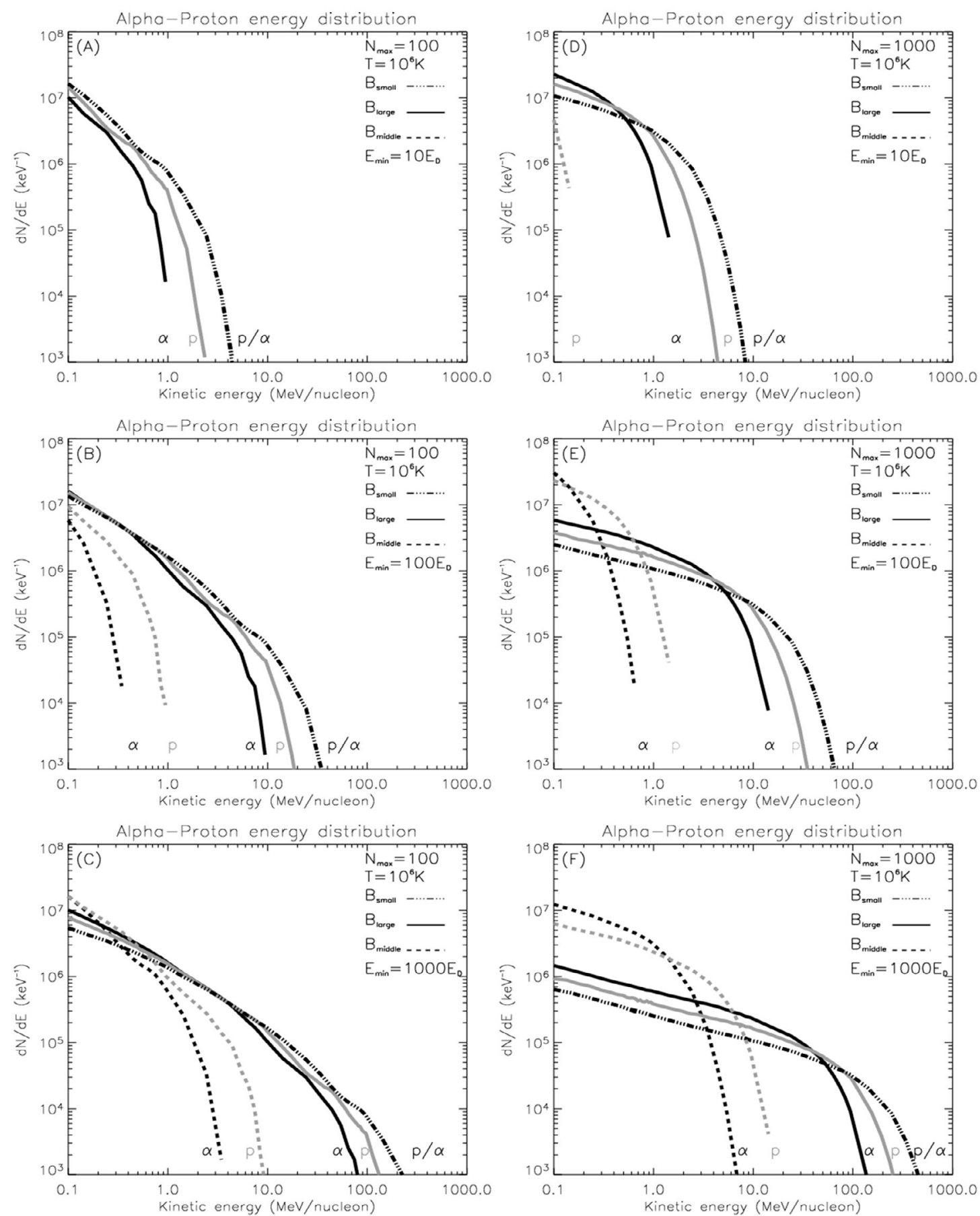

Fig. 7. The same as Fig. 4, but for the case of alpha particles. Note that the grey color corresponds to the proton distribution. Proton and alpha particles distributions are the same in the case $B_{\text {small }}$.

energy distributions for electrons and protons lies in the low energy part where the effects of cut-offs from electron and proton energy distributions in specific RCS configurations clearly appear.

\subsection{Final alpha particle and heavier ion kinetic energy distributions}

As shown in Sect. 3, the energy gain for ions is a function of the parameter $Z / A$ that implies that the energy distributions will be similar for all particle species with the same $Z / A$. Therefore, only alpha particle distributions are shown in the present paper, but similar conclusions could be drawn for the heavier $\gamma$-ray line producing ions such as ${ }^{12} \mathrm{C},{ }^{16} \mathrm{O},{ }^{22} \mathrm{Ne},{ }^{24} \mathrm{Mg}$, and ${ }^{28} \mathrm{Si}$. Figure 7 shows the distribution in energy per nucleon for alpha particles when compared to the proton energy distributions. The energy gain of alpha particles in the different configurations is given by Eqs. (17), (26), and (40). As expected, Fig. 7 shows that the energy gain in $\mathrm{MeV} /$ nucleon is independent of the ratio $Z / A$ in the case of small longitudinal magnetic fields, while it varies as $Z / A$ and as $(Z / A)^{3 / 2}$ in the case of, respectively, large and intermediate values of the magnetic field. In these last two cases, the lower energy gain for alpha particles leads to a spectrum steeper and with a lower energy cut-off than for protons. This implies that for these configurations, the production of $\gamma$-ray lines will be mostly produced by protons with a small contribution of alpha particles. 
Table 1. Number of accelerated electrons above $20 \mathrm{keV}$ and $300 \mathrm{keV}$ for a total volume of distributed RCS of $1 \operatorname{arcsec}^{3}$.

\begin{tabular}{c|cccc|cccc}
\hline \hline & \multicolumn{4}{|c|}{$N_{\mathrm{e}}>20 \mathrm{keV}$} & \multicolumn{4}{c}{$N_{\mathrm{e}}>300 \mathrm{keV}$} \\
\hline$N_{\max }=100$ & $B_{\text {small }}$ & $B_{\text {middle }}$ & $B_{\text {large }}$ & $B_{\text {total }}$ & $B_{\text {small }}$ & $B_{\text {middle }}$ & $B_{\text {large }}$ & $B_{\text {total }}$ \\
\hline$E_{\min }=10 E_{\mathrm{D}}$ & 0 & $2.1 \times 10^{33}$ & $1.7 \times 10^{33}$ & $3.8 \times 10^{33}$ & 0 & $5.5 \times 10^{32}$ & $3.1 \times 10^{32}$ & $8.7 \times 10^{32}$ \\
$E_{\min }=100 E_{\mathrm{D}}$ & $7.3 \times 10^{29}$ & $3.1 \times 10^{33}$ & $2.9 \times 10^{33}$ & $6.0 \times 10^{33}$ & 0 & $1.9 \times 10^{33}$ & $1.5 \times 10^{33}$ & $3.3 \times 10^{33}$ \\
$E_{\min }=1000 E_{\mathrm{D}}$ & $4.8 \times 10^{32}$ & $3.5 \times 10^{33}$ & $3.4 \times 10^{33}$ & $7.4 \times 10^{33}$ & $1.9 \times 10^{28}$ & $3.0 \times 10^{33}$ & $2.7 \times 10^{33}$ & $5.7 \times 10^{33}$ \\
\hline$N_{\max }=1000$ & $B_{\text {small }}$ & $B_{\text {middle }}$ & $B_{\text {large }}$ & $B_{\text {total }}$ & $B_{\text {small }}$ & $B_{\text {middle }}$ & $B_{\text {large }}$ & $B_{\text {total }}$ \\
\hline$E_{\min }=10 E_{\mathrm{D}}$ & 0 & $3.4 \times 10^{33}$ & $3.2 \times 10^{33}$ & $6.6 \times 10^{33}$ & 0 & $2.3 \times 10^{33}$ & $1.7 \times 10^{33}$ & $4.1 \times 10^{33}$ \\
$E_{\min }=100 E_{\mathrm{D}}$ & $7.3 \times 10^{31}$ & $3.6 \times 10^{33}$ & $3.5 \times 10^{33}$ & $7.1 \times 10^{33}$ & 0 & $3.3 \times 10^{33}$ & $3.1 \times 10^{33}$ & $6.4 \times 10^{33}$ \\
$E_{\min }=1000 E_{\mathrm{D}}$ & $2.2 \times 10^{33}$ & $3.6 \times 10^{33}$ & $3.6 \times 10^{33}$ & $9.4 \times 10^{33}$ & $8.8 \times 10^{30}$ & $2.5 \times 10^{33}$ & $3.5 \times 10^{33}$ & $6.0 \times 10^{33}$ \\
\hline
\end{tabular}

Table 2. Number of accelerated protons above $1 \mathrm{MeV}, 30 \mathrm{MeV}$, and $300 \mathrm{MeV}$ for a total volume of distributed RCS of 1 arcsec ${ }^{3}$.

\begin{tabular}{c|cccc|cccc}
\hline \hline & \multicolumn{4}{|c|}{$N_{\mathrm{p}}>1 \mathrm{MeV}$} & \multicolumn{4}{c}{$N_{\mathrm{p}}>30 \mathrm{MeV}$} \\
\hline$N_{\max }=100$ & $B_{\text {small }}$ & $B_{\text {middle }}$ & $B_{\text {large }}$ & $B_{\text {total }}$ & $B_{\text {small }}$ & $B_{\text {middle }}$ & $B_{\text {large }}$ & $B_{\text {total }}$ \\
\hline$E_{\min }=10 E_{\mathrm{D}}$ & $1.5 \times 10^{32}$ & 0 & $2.8 \times 10^{31}$ & $1.9 \times 10^{32}$ & 0 & 0 & 0 & 0 \\
$E_{\min }=100 E_{\mathrm{D}}$ & $1.2 \times 10^{33}$ & $7.6 \times 10^{28}$ & $7.4 \times 10^{32}$ & $1.9 \times 10^{33}$ & $4.7 \times 10^{30}$ & 0 & $3.8 \times 10^{27}$ & $4.7 \times 10^{30}$ \\
$E_{\min }=1000 E_{\mathrm{D}}$ & $2.5 \times 10^{33}$ & $4.1 \times 10^{32}$ & $2.1 \times 10^{33}$ & $5.0 \times 10^{33}$ & $5.6 \times 10^{32}$ & 0 & $3.1 \times 10^{32}$ & $8.8 \times 10^{32}$ \\
\hline$N_{\max }=1000$ & $B_{\text {small }}$ & $B_{\text {middle }}$ & $B_{\text {large }}$ & $B_{\text {total }}$ & $B_{\text {small }}$ & $B_{\text {middle }}$ & $B_{\text {large }}$ & $B_{\text {total }}$ \\
\hline$E_{\min }=10 E_{\mathrm{D}}$ & $1.2 \times 10^{33}$ & 0 & $4.3 \times 10^{32}$ & $1.6 \times 10^{33}$ & 0 & 0 & 0 & 0 \\
$E_{\min }=100 E_{\mathrm{D}}$ & $3.0 \times 10^{33}$ & $2.5 \times 10^{31}$ & $2.6 \times 10^{33}$ & $5.6 \times 10^{33}$ & $1.6 \times 10^{32}$ & 0 & $4.7 \times 10^{30}$ & $1.7 \times 10^{32}$ \\
$E_{\min }=1000 E_{\mathrm{D}}$ & $1.2 \times 10^{33}$ & $2.5 \times 10^{31}$ & $2.6 \times 10^{33}$ & $5.6 \times 10^{33}$ & $8.8 \times 10^{30}$ & $2.5 \times 10^{33}$ & $3.5 \times 10^{33}$ & $6.0 \times 10^{33}$ \\
\hline
\end{tabular}

\begin{tabular}{c|cccc}
\hline \hline & \multicolumn{4}{|c}{$N_{\mathrm{p}}>300 \mathrm{MeV}$} \\
\hline$N_{\max }=100$ & $B_{\text {small }}$ & $B_{\text {middle }}$ & $B_{\text {large }}$ & $B_{\text {total }}$ \\
\hline$E_{\min }=10 E_{\mathrm{D}}$ & 0 & 0 & 0 & 0 \\
$E_{\min }=100 E_{\mathrm{D}}$ & 0 & 0 & 0 & 0 \\
$E_{\min }=1000 E_{\mathrm{D}}$ & $4.8 \times 10^{30}$ & 0 & $2.2 \times 10^{28}$ & $4.9 \times 10^{30}$ \\
\hline$N_{\max }=1000$ & $B_{\text {small }}$ & $B_{\text {middle }}$ & $B_{\text {large }}$ & $B_{\text {total }}$ \\
\hline$E_{\min }=10 E_{\mathrm{D}}$ & 0 & 0 & 0 & 0 \\
$E_{\min }=100 E_{\mathrm{D}}$ & 0 & 0 & 0 & 0 \\
$E_{\min }=1000 E_{\mathrm{D}}$ & 0 & $3.4 \times 10^{33}$ & $3.2 \times 10^{33}$ & $6.6 \times 10^{33}$ \\
\hline
\end{tabular}

\subsection{Electron and proton numbers - Energy content}

Electron and proton numbers for energies above $20 \mathrm{keV}$ and $300 \mathrm{keV}$ for electrons and above $1 \mathrm{MeV}, 30 \mathrm{MeV}$, and $300 \mathrm{MeV}$ for protons are computed from the energy distributions. The different results reported in Tables 1 and 2 are obtained for a total volume of distributed RCS of $1 \operatorname{arcsec}^{3}$. Electron numbers are not strongly different in the cases $B_{\text {middle }}, B_{\text {large }}$, and $B_{\text {total }}$ (sum of all other distributions) compared to the values found in the case $B_{\text {small }}$, in which electrons are not efficiently accelerated especially at high energies. In the case of protons, the cases $B_{\text {small }}$, $B_{\text {large, }}$, and $B_{\text {total }}$ lead to comparable number of protons accelerated above $1 \mathrm{MeV}, 30 \mathrm{MeV}$, and $300 \mathrm{MeV}$. In the case of an intermediate configuration, a small number of protons is accelerated above $1 \mathrm{MeV}$ and thus the production of $\gamma$-ray lines will be negligible in that case.

Figure 8 presents the total energy contained by electrons above $20 \mathrm{keV}$ and by protons above $1 \mathrm{MeV}$ for a total volume of distributed RCS of $1 \operatorname{arcsec}^{3}$ for the different cases. Depending on the characteristics of the RCS, the energy is roughly equipartitioned between electrons and ions or preferentially go to electrons or ions. This will be discussed in the following section with respect to observations.

\section{Hard X-ray and gamma ray fluxes}

We now compute the production of X-ray and $\gamma$-ray line fluxes produced by the accelerated particles in the dense layers of the solar atmosphere (thick target approximation) after free propagation from the active region (see the sketch of the model in the introduction). In the most simple thick target approximation (see, e.g., Brown 1971), the number of X-ray photons of energy $h v$ produced in a homogeneous medium of density $\mathrm{n}$ by an electron of initial energy $E_{0}$ is:

$\mu\left(h v, E_{\mathrm{c} 0}\right)=-\int_{h v}^{E_{\mathrm{c} 0}} \sigma\left(h v, E_{\mathrm{c}}\right) n v\left(E_{\mathrm{c}}\right) \frac{\mathrm{d} t}{\mathrm{~d} E_{\mathrm{c}}} \mathrm{d} E_{\mathrm{c}}$,

where $E_{\mathrm{c}}$ is the particle kinetic energy, $\sigma\left(h v, E_{\mathrm{c}}\right)$ is the $\mathrm{X}$ ray bremstrahlung cross-section, $v\left(E_{\mathrm{c}}\right)$ is the velocity of the particle, and $\mathrm{d} E_{\mathrm{c}} / \mathrm{d} t$ is the energy loss due to collisions. In the case of electrons, the Coulomb collision energy losses are given by (Trubnikov 1965):

$\frac{\mathrm{d} E_{\mathrm{c}}}{\mathrm{d} t}=\left\{\begin{array}{l}-4.9 \times 10^{-9} E_{\mathrm{c}}^{-1 / 2} n\left(\mathrm{keV} \mathrm{s}^{-1}\right) \text { for } E_{\mathrm{c}} \leq 160 \mathrm{keV} . \\ -3.8 \times 10^{-10} n\left(\mathrm{keV} \mathrm{s}^{-1}\right) \text { for } E_{\mathrm{c}}>160 \mathrm{keV} .\end{array}\right.$

The X-ray flux produced at photon energy $h v$ is then given by:

$I(h v)=\int_{E_{\mathrm{c} 0}}^{+\infty} F\left(E_{\mathrm{c} 0}\right) \mu\left(h v, E_{\mathrm{c} 0}\right) \mathrm{d} E_{\mathrm{c} 0}$ 


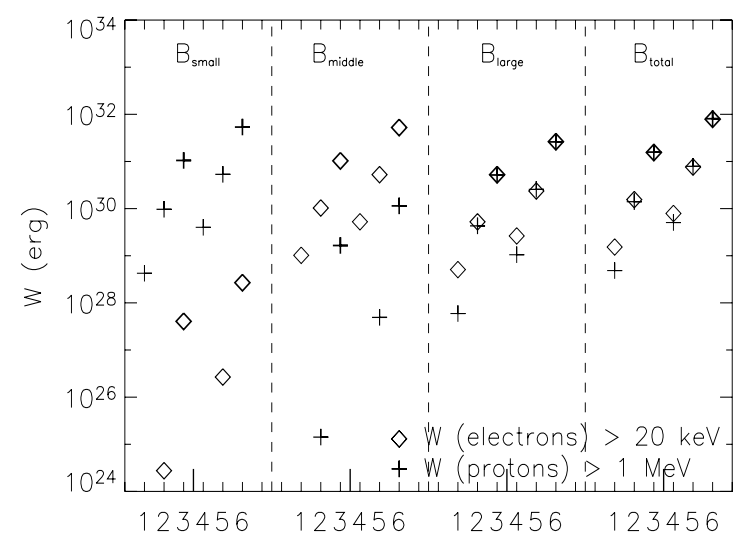

Fig. 8. Energy contained in electrons above $20 \mathrm{keV}$ (cross marks) and in protons above $1 \mathrm{MeV}$ (diamond marks) as a function of the electric field normalizations, number of interactions, and magnetic field configuration. The values reported in this figure are plotted in four series corresponding to the four magnetic field configurations, respectively, from left to right, $B_{\text {small }}, B_{\text {middle }}, B_{\text {large }}$, and $B_{\text {total }}$. For each magnetic field configuration, the cases 1,2 , and 3 correspond to $N_{\max }=100$ and $E_{\min }=10 E_{\mathrm{D}}, E_{\min }=100 E_{\mathrm{D}}$, and $E_{\min }=1000 E_{\mathrm{D}}$. The cases 4,5 and 6 correspond to $N_{\max }=1000$ and $E_{\min }=10 E_{\mathrm{D}}, E_{\min }=100 E_{\mathrm{D}}$, and $E_{\min }=1000 E_{\mathrm{D}}$.

where $F\left(E_{\mathrm{c} 0}\right)$ is the energy distribution of accelerated electrons. For gamma-ray line productions, the computation of the gamma ray flux is done in a similar way by replacing the bremsstrahlung $\mathrm{X}$-ray producing cross section by the gamma ray line producing cross sections. Proton energy losses are due to excitation and ionization of the ambient hydrogen atoms and are given by (Jackson 1962):

$\frac{\mathrm{d} E_{\mathrm{c}}}{\mathrm{d} t}=\left\{\begin{array}{l}-1.65 \times 10^{-12} E_{\mathrm{c}}^{-1 / 3} n\left(\mathrm{MeV} \mathrm{s}^{-1}\right) \text { for } E_{\mathrm{c}} \leq 560 \mathrm{MeV} . \\ -2 \times 10^{-13} n\left(\mathrm{MeV} \mathrm{s}^{-1}\right) \text { for } E_{\mathrm{c}}>560 \mathrm{MeV}\end{array}\right.$

In the case of ions, energy losses are those of protons multiplied by a factor $Z^{2} / A$, where $Z$ is the atomic number of the element considered and $\mathrm{A}$ is its mass number. In the following, the strong de-excitation lines at $6.129 \mathrm{MeV}$ from ${ }^{16} \mathrm{O}, 4.438 \mathrm{MeV}$ from the first excited state of ${ }^{12} \mathrm{C}$ populated directly and by the spallation reactions on ${ }^{16} \mathrm{O}, 1.779 \mathrm{MeV}$ from ${ }^{28} \mathrm{Si}, 1.634 \mathrm{MeV}$ from ${ }^{20} \mathrm{Ne}$, $1.369 \mathrm{MeV}$ from ${ }^{24} \mathrm{Mg}$ are computed. The thick-target production of the broad $\gamma$-ray lines from interaction of the energetic $\mathrm{C}$, $\mathrm{O}, \mathrm{Ne}, \mathrm{Mg}$, and $\mathrm{Si}$ with the ambient hydrogen or helium nuclei is also computed.

\subsection{Hard X-ray spectra}

Figure 9 shows the different hard X-ray spectra calculated as indicated above from the energy distributions of electrons shown in Fig. 5. In most cases (b, c, e, f), flat and even spectra increasing with energy are observed at low energies for $B_{\text {large }}$ and $B_{\text {middle }}$ configurations. These kinds of photon spectra are not realistic and result from the very flat shape of the electron spectrum at X-ray producing energies (see Figs. 5b, c, e, f). Let us recall that these flat electron spectra are linked to flatness of the slope of the distribution of the magnetic energy release (see Fig. 3). The decreasing part of the X-ray spectrum is well fitted by a double power law with slopes indicated on the figures. In the $B_{\text {small }}$ configuration, X-ray emission is not efficiently produced and is observed only in the case of a large normalization of the electric field to the Dreicer field. The comparison of the predicted spectral indices with respect to observed ones will be discussed in the next section.

\subsection{Gamma ray line productions}

Table 3 gives the values of the fluences for different excitation lines produced by energetic protons, in the case of different configurations of the RCS and number of interactions. In this table, the relative fluences are computed assuming relative abundances of 1 for all species. In these conditions the results show a combination of the effect of the energy dependence of the different cross sections and of the variation with energy of particle spectra. For a given accelerated proton spectrum, the relative efficiency for producing $\gamma$-ray lines is systematically decreasing from the $\gamma$-ray lines of $\mathrm{Mg}$ at $1.369 \mathrm{MeV}$, of $\mathrm{Ne}$ at $1.63 \mathrm{MeV}$, of $\mathrm{C}$ at $4.438 \mathrm{MeV}$ produced by direct excitation, and of $\mathrm{O}$ at $6.129 \mathrm{MeV}$. This is consistent with the energy dependence of nuclear cross sections. The fluence of the de-excitation line of ${ }^{12} \mathrm{C}$ at $4.438 \mathrm{MeV}$ resulting from spallation on ${ }^{16} \mathrm{O}$ and computed with $\mathrm{C} / \mathrm{O}=1$ is larger than the fluence of ${ }^{16} \mathrm{O}$ at $6.129 \mathrm{MeV}$ in the case of large values for the normalization of the electric field. This reflects a combination of the larger values of the cross section of the spallation reaction in the $10-100 \mathrm{MeV}$ range and of the larger number of protons in this energy range in the case of large values of $E_{\min }$. The table also reflects that the relative efficiency for producing $\gamma$-ray lines is linked to the number and spectrum of energetic protons in the few $\mathrm{MeV}$ range. The configuration with $B_{\text {small }}$ is obviously the most efficient one and the production of $\gamma$-ray lines in the $B_{\text {middle }}$ configuration very inefficient.

In the same conditions, Table 4 gives the fluences of the same $\gamma$-ray lines when produced by energetic $\alpha$ particles. Table 4 reflects the same trends as Table 3 for the production of the different $\gamma$-ray lines and for the dependency on the RCS configurations except for the lines of ${ }^{24} \mathrm{Mg}$ at $1.369 \mathrm{MeV}$ and of ${ }^{20} \mathrm{Ne}$ at $1.634 \mathrm{MeV}$. The fluence of the neon line is indeed larger than the fluence of the magnesium line in that case. This is related to the larger energy band of alpha particles producing the neon when compared to the ones producing the magnesium line. The comparison of fluences of $\gamma$-ray lines produced by energetic $\alpha$ particles with the ones produced by energetic protons reflects a combination of the steeper spectrum for accelerated $\alpha$ particles and of the cross sections. Apart from the production of the ${ }^{12} \mathrm{C}$ line from spallation on ${ }^{16} \mathrm{O}$, the fluences due to alpha particles are smaller, the difference being even larger for the neon and magnesium lines for which the cross sections are smaller for alpha particles than for protons. The relative production of the ${ }^{12} \mathrm{C}$ line from spallation on ${ }^{16} \mathrm{O}$ is larger for alpha particles than for protons. In the case of alpha particles, the cross section is indeed sensitive to particles between 4 to $10 \mathrm{MeV} /$ nuc, while for protons the cross sections begins to be significant for protons around $20 \mathrm{MeV}$ that are less efficiently produced than alpha particles below $10 \mathrm{MeV} /$ nuc (see Figs. 7b, c, e, f).

Table 5 gives the fluences of the same $\gamma$-ray lines for the inverse reactions, i.e., the broad $\gamma$-ray lines produced by the interaction of energetic $\mathrm{Mg}, \mathrm{Ne}, \mathrm{C}$, and $\mathrm{O}$, respectively, on ambient $\mathrm{H}$. The values are still obtained for relative abundances of the different accelerated species equal to 1 . The cross sections of the inverse and direct reactions in $\mathrm{MeV} /$ nuc are the same. In these conditions, the differences between the results of Tables 3 and 5 come from the difference in proton and ion spectra and in the energy losses that are multiplied by a factor $Z^{2} / A$ for ions. Since the production rate of photons in a thick target approximation is proportional to the inverse of the energy losses, heavier ions are expected to produce $A / Z^{2}$ fewer $\gamma$-ray photons than protons in the case of similar cross sections. Moreover, Fig. 7 shows that the relative efficiency for producing energetic ions with respect 

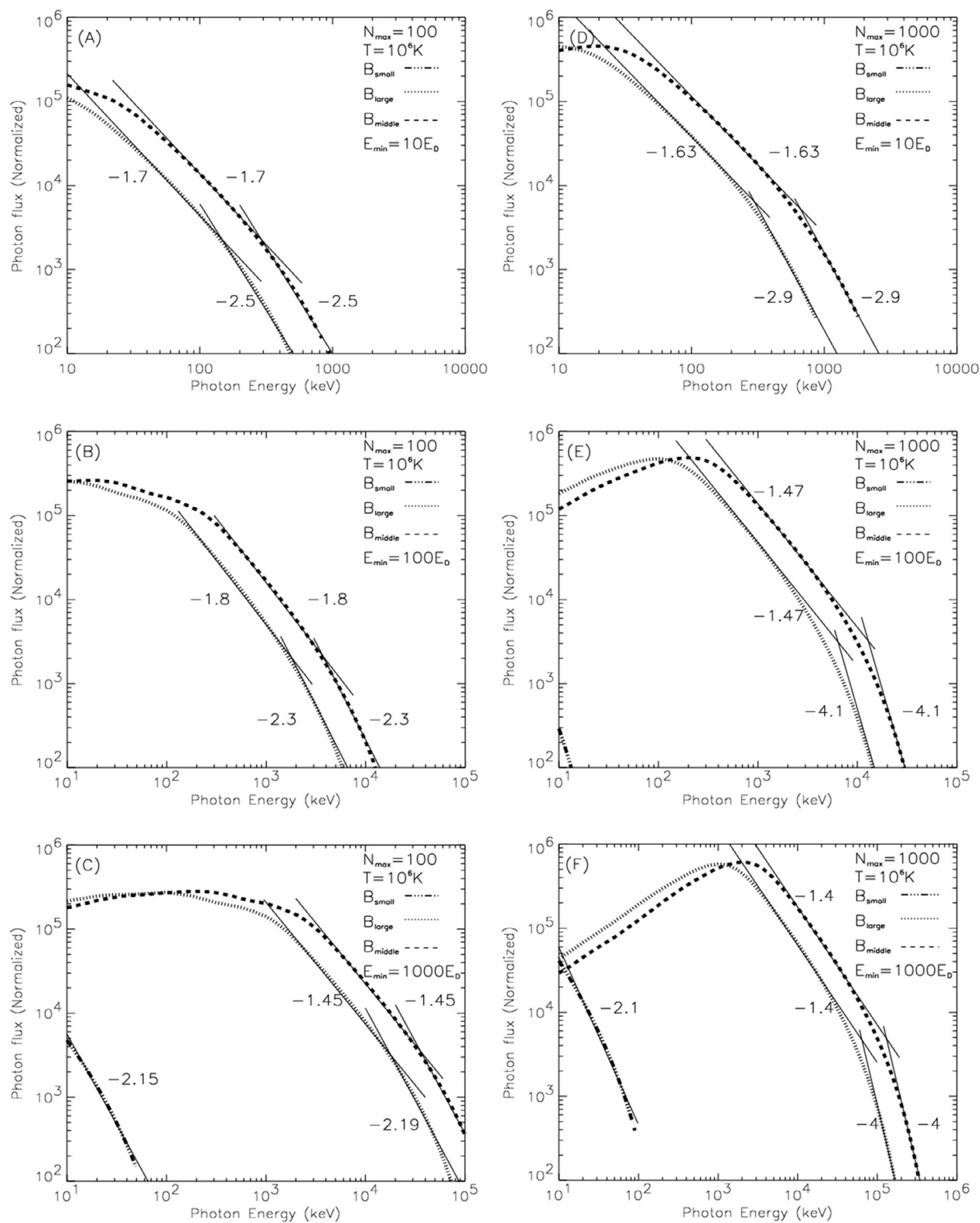

Fig. 9. Photon energy distributions calculated from the electron energy distributions presented in Fig. 5. In each panel, the dashed-dotted line corresponds to the distribution in the case $B_{\text {small }}$, the dashed line corresponds to the case $B_{\text {middle }}$, and the dotted line corresponds to the case $B_{\text {large }}$. The values of the spectral index are determined by a linear fit.

to protons is smaller for $B_{\text {large }}$ than for $B_{\text {small }}$. This is why the production of inverse $\gamma$-ray lines in the case of $B_{\text {large }}$ is smaller (and sometimes negligible) when compared to the production of direct lines. In the case of $B_{\text {small }}$, the lower values of the $\gamma$-ray line fluences for the inverse reactions are only due to the effect of the energy losses. In the case $B_{\text {large }}$ and $B_{\text {middle }}$, the lower efficiency for photon production of the inverse reactions is a combination of spectra and energy loss effects.

Table 6 gives the fluences of the broad $\gamma$-ray lines produced by the interaction of energetic $\mathrm{Mg}, \mathrm{Ne}, \mathrm{C}$, and $\mathrm{O}$ on ambient ${ }^{4} \mathrm{He}$. The values are still obtained for the same abundances of the different species equal to 1 . The differences with the values reported in Table 4 come only from the energy losses. As expected, the $\gamma$-ray line fluences produced by the inverse reactions are lower since the ion energy losses are more important.

Figure 10 shows the ratio of the different $\gamma$-ray line fluences observed from the carbon line at $4.438 \mathrm{MeV}$, the silicium line at $1.779 \mathrm{MeV}$, the neon line at $1.634 \mathrm{MeV}$, and the magnesium line at $1.369 \mathrm{MeV}$ with respect to the fluence of the Oxygen line at 6.129 MeV. The fluences are computed here for the direct production of the lines from both accelerated proton and alpha particles and using either coronal or photospheric abundances for 
Table 3. Gamma ray lines fluences produced by accelerated protons (relative units).

\begin{tabular}{c|ccc|ccc}
\hline \hline & \multicolumn{3}{|c|}{$1.3639 \mathrm{MeV}$} & \multicolumn{3}{c}{$1.634 \mathrm{MeV}$} \\
\hline$N_{\max }=100$ & $B_{\text {small }}$ & $B_{\text {middle }}$ & $B_{\text {large }}$ & $B_{\text {small }}$ & $B_{\text {middle }}$ & $B_{\text {large }}$ \\
\hline$E_{\min }=100 E_{\mathrm{D}}$ & $1.5 \times 10^{4}$ & 0 & $3.1 \times 10^{3}$ & $10^{4}$ & 0 & $2.5 \times 10^{3}$ \\
$E_{\min }=1000 E_{\mathrm{D}}$ & $2.7 \times 10^{5}$ & $2.4 \times 10^{2}$ & $1.4 \times 10^{5}$ & $1.6 \times 10^{5}$ & $2.3 \times 10^{2}$ & $7.8 \times 10^{4}$ \\
\hline$N_{\max }=1000$ & $B_{\text {small }}$ & $B_{\text {middle }}$ & $B_{\text {large }}$ & $B_{\text {small }}$ & $B_{\text {middle }}$ & $B_{\text {large }}$ \\
\hline$E_{\min }=100 E_{\mathrm{D}}$ & $1.3 \times 10^{5}$ & 0 & $3.9 \times 10^{4}$ & $8.2 \times 10^{4}$ & 0 & $2.8 \times 10^{4}$ \\
$E_{\min }=1000 E_{\mathrm{D}}$ & $1.4 \times 10^{6}$ & $5.3 \times 10^{3}$ & $8.2 \times 10^{5}$ & $8.7 \times 10^{5}$ & $4.5 \times 10^{3}$ & $4.7 \times 10^{5}$ \\
\hline
\end{tabular}

\begin{tabular}{c|ccc|ccc|ccc}
\hline \hline & \multicolumn{3}{|c|}{$4.438 \mathrm{MeV}(\mathrm{C})$} & \multicolumn{3}{c|}{$4.438 \mathrm{MeV}(\mathrm{O})$} & \multicolumn{3}{c}{$6.129 \mathrm{MeV}$} \\
\hline$N_{\max }=100$ & $B_{\text {small }}$ & $B_{\text {middle }}$ & $B_{\text {large }}$ & $B_{\text {small }}$ & $B_{\text {middle }}$ & $B_{\text {large }}$ & $B_{\text {small }}$ & $B_{\text {middle }}$ & $B_{\text {large }}$ \\
\hline$E_{\min }=100 E_{\mathrm{D}}$ & $7.5 \times 10^{3}$ & 0 & $1.2 \times 10^{3}$ & $1.1 \times 10^{3}$ & 0 & 0 & $3.3 \times 10^{3}$ & 0 & $3.2 \times 10^{2}$ \\
$E_{\min }=1000 E_{\mathrm{D}}$ & $1.2 \times 10^{5}$ & 39 & $6.5 \times 10^{4}$ & $9.3 \times 10^{4}$ & 0 & $4.3 \times 10^{4}$ & $8.1 \times 10^{4}$ & 0 & $4.0 \times 10^{4}$ \\
\hline$N_{\max }=1000$ & $B_{\text {small }}$ & $B_{\text {middle }}$ & $B_{\text {large }}$ & $B_{\text {small }}$ & $B_{\text {middle }}$ & $B_{\text {large }}$ & $B_{\text {small }}$ & $B_{\text {middle }}$ & $B_{\text {large }}$ \\
\hline$E_{\min }=100 E_{\mathrm{D}}$ & $7.0 \times 10^{4}$ & 0 & $1.8 \times 10^{4}$ & $2.3 \times 10^{4}$ & 0 & $1.5 \times 10^{3}$ & $3.6 \times 10^{4}$ & 0 & $7.2 \times 10^{3}$ \\
$E_{\min }=1000 E_{\mathrm{D}}$ & $5.5 \times 10^{5}$ & $1.5 \times 10^{3}$ & $3.6 \times 10^{5}$ & $5.3 \times 10^{5}$ & 0 & $3.1 \times 10^{5}$ & $4.1 \times 10^{5}$ & $2.9 \times 10^{2}$ & $2.5 \times 10^{5}$ \\
\hline
\end{tabular}

Table 4. Gamma ray lines fluences produced by accelerated alpha particles (relative units).

\begin{tabular}{c|ccc|ccc}
\hline \hline & \multicolumn{3}{|c|}{$1.3639 \mathrm{MeV}$} & \multicolumn{3}{c}{$1.634 \mathrm{MeV}$} \\
\hline$N_{\max }=100$ & $B_{\text {small }}$ & $B_{\text {middle }}$ & $B_{\text {large }}$ & $B_{\text {small }}$ & $B_{\text {middle }}$ & $B_{\text {large }}$ \\
\hline$E_{\min }=100 E_{\mathrm{D}}$ & $6.6 \times 10^{3}$ & 0 & $7.6 \times 10^{2}$ & $9.2 \times 10^{3}$ & 0 & $8.2 \times 10^{2}$ \\
$E_{\min }=1000 E_{\mathrm{D}}$ & $2.9 \times 10^{4}$ & 0 & $1.4 \times 10^{4}$ & $5.4 \times 10^{4}$ & 0 & $2.3 \times 10^{4}$ \\
\hline$N_{\max }=1000$ & $B_{\text {small }}$ & $B_{\text {middle }}$ & $B_{\text {large }}$ & $B_{\text {small }}$ & $B_{\text {middle }}$ & $B_{\text {large }}$ \\
\hline$E_{\min }=100 E_{\mathrm{D}}$ & $3.5 \times 10^{4}$ & 0 & $8.1 \times 10^{3}$ & $5.8 \times 10^{4}$ & 0 & $9.0 \times 10^{3}$ \\
$E_{\min }=1000 E_{\mathrm{D}}$ & $6.1 \times 10^{4}$ & $4.1 \times 10^{2}$ & $4.9 \times 10^{4}$ & $1.4 \times 10^{5}$ & $4.2 \times 10^{2}$ & $1.0 \times 10^{5}$ \\
\hline
\end{tabular}

\begin{tabular}{c|ccc|ccc|ccc}
\hline \hline & \multicolumn{3}{|c|}{$4.438 \mathrm{MeV}(\mathrm{C})$} & \multicolumn{3}{c|}{$4.438 \mathrm{MeV}(\mathrm{O})$} & \multicolumn{3}{c}{$6.129 \mathrm{MeV}$} \\
\hline$N_{\max }=100$ & $B_{\text {small }}$ & $B_{\text {middle }}$ & $B_{\text {large }}$ & $B_{\text {small }}$ & $B_{\text {middle }}$ & $B_{\text {large }}$ & $B_{\text {small }}$ & $B_{\text {middle }}$ & $B_{\text {large }}$ \\
\hline$E_{\min }=100 E_{\mathrm{D}}$ & $7.1 \times 10^{3}$ & 0 & $2.8 \times 10^{2}$ & $5.9 \times 10^{3}$ & 0 & 80 & $1.8 \times 10^{3}$ & 0 & 20 \\
$E_{\min }=1000 E_{\mathrm{D}}$ & $5.6 \times 10^{4}$ & 0 & $2.1 \times 10^{4}$ & $4.6 \times 10^{4}$ & 0 & $1.7 \times 10^{4}$ & $1.4 \times 10^{4}$ & 0 & $5.3 \times 10^{3}$ \\
\hline$N_{\max }=1000$ & $B_{\text {small }}$ & $B_{\text {middle }}$ & $B_{\text {large }}$ & $B_{\text {small }}$ & $B_{\text {middle }}$ & $B_{\text {large }}$ & $B_{\text {small }}$ & $B_{\text {middle }}$ & $B_{\text {large }}$ \\
\hline$E_{\min }=100 E_{\mathrm{D}}$ & $5.1 \times 10^{4}$ & 0 & $5.0 \times 10^{3}$ & $4.5 \times 10^{4}$ & 0 & $2.4 \times 10^{3}$ & $1.4 \times 10^{4}$ & 0 & $7.3 \times 10^{2}$ \\
$E_{\min }=1000 E_{\mathrm{D}}$ & $1.6 \times 10^{5}$ & 20 & $1.0 \times 10^{5}$ & $1.3 \times 10^{5}$ & 0 & $8.8 \times 10^{4}$ & $3.8 \times 10^{4}$ & 0 & $2.7 \times 10^{4}$ \\
\hline
\end{tabular}

the ambient medium (see Table 7 from Murphy et al. 1991). We consider that particle acceleration occurs in the corona, hence we choose a coronal value of 0.097 for the ratio $\mathrm{He} / \mathrm{H}$ from which we accelerate particles. This implies that the contribution of alpha particles to the $\gamma$-ray line production will thus be limited, since $\alpha / \mathrm{p}$ will be of the order of 0.1 . The fluences are computed in the case $B_{\text {total }}$ for two larger electric field normalizations $E_{\text {min }}=100 E_{\mathrm{D}}$ and $E_{\min }=1000 E_{\mathrm{D}}$ and for two different maximum numbers of interactions $N_{\max }=100$ and $N_{\max }=1000$. The average of the different values of the $\gamma$-ray line ratio for both photosphere and coronal values are indicated. The interval of accessible values for different electric field normalizations and interaction numbers is indicated by both abundances. We also indicated the values of the average ratio of $\gamma$-ray lines deduced from SMM observations by Share \& Murphy (1995) that have measured the fluxes of ten narrow gamma ray lines for $19 \mathrm{X}$ class flares. The lines around these values represent the dispersion of the ratio for the 19 flares. We also report the $\gamma$-ray line ratio obtained from the RHESSI data during the 23 July 2002 flare (Smith et al. 2003) in this figure. Comparison of the gamma ray line ratio predicted by the model to the observed ones shows that the ratio of the lines are well reproduced except for the neon line. $\mathrm{The} \mathrm{Mg} / \mathrm{O}$ and $\mathrm{Si} / \mathrm{O}$ ratios are correctly reproduced for coronal abundance in good consistency with the results of Share \& Murphy (1995). The observed C/O ratio is also better reproduced for a coronal abundance. However, the ratio Ne/O determined from the model is underestimated compared to the observed values. This is in agreement with the well-known problem of the intense production of the neon line in flares.

\section{Discussion and conclusion}

In the previous sections we have presented the results of the simulation of electron and ion acceleration in flares based on a CA model to mimic the energy release process in the active region and on acceleration by a distribution of super Dreicer direct electric fields in simple geometrical configurations of reconnecting current sheets (RCS). By considering three different assumptions for the longitudinal magnetic field in the RCS (i.e., no longitudinal magnetic field, large or intermediate values of the longitudinal magnetic fields allowing to magnetize electrons and ions, or mostly electrons), we obtain three different values of particle energy gains in the RCS for electrons, protons, and heavier ions. The kinetic energy distributions of electrons, protons, and heavier ions are computed for three different values of the normalization of the electric field with respect 
Table 5. Gamma ray lines fluences produced by inverse reactions of energetic $\mathrm{Ne}, \mathrm{Mg}, \mathrm{C}$, and $\mathrm{O}$ ions on ambiant hydrogen.

\begin{tabular}{c|ccc|ccc}
\hline \hline & \multicolumn{3}{|c|}{$1.3639 \mathrm{MeV}$} & \multicolumn{3}{c}{$1.634 \mathrm{MeV}$} \\
\hline$N_{\max }=100$ & $B_{\text {small }}$ & $B_{\text {middle }}$ & $B_{\text {large }}$ & $B_{\text {small }}$ & $B_{\text {middle }}$ & $B_{\text {large }}$ \\
\hline$E_{\min }=100 E_{\mathrm{D}}$ & $7.1 \times 10^{3}$ & 0 & $1.6 \times 10^{2}$ & $5.5 \times 10^{3}$ & 0 & $1.7 \times 10^{2}$ \\
$E_{\min }=1000 E_{\mathrm{D}}$ & $1.1 \times 10^{5}$ & 0 & $3.0 \times 10^{4}$ & $7.7 \times 10^{4}$ & 0 & $2.0 \times 10^{4}$ \\
\hline$N_{\max }=1000$ & $B_{\text {small }}$ & $B_{\text {middle }}$ & $B_{\text {large }}$ & $B_{\text {small }}$ & $B_{\text {middle }}$ & $B_{\text {large }}$ \\
\hline$E_{\min }=100 E_{\mathrm{D}}$ & $6.3 \times 10^{4}$ & 0 & $3.3 \times 10^{3}$ & $4.5 \times 10^{4}$ & 0 & $3.1 \times 10^{3}$ \\
$E_{\min }=1000 E_{\mathrm{D}}$ & $5.2 \times 10^{5}$ & 21 & $1.9 \times 10^{5}$ & $3.7 \times 10^{5}$ & 34 & $1.2 \times 10^{5}$ \\
\hline
\end{tabular}

\begin{tabular}{c|ccc|ccc|ccc}
\hline \hline & \multicolumn{3}{|c|}{$4.438 \mathrm{MeV}(\mathrm{C})$} & \multicolumn{3}{c|}{$4.438 \mathrm{MeV}(\mathrm{O})$} & \multicolumn{3}{c}{$6.129 \mathrm{MeV}$} \\
\hline$N_{\max }=100$ & $B_{\text {small }}$ & $B_{\text {middle }}$ & $B_{\text {large }}$ & $B_{\text {small }}$ & $B_{\text {middle }}$ & $B_{\text {large }}$ & $B_{\text {small }}$ & $B_{\text {middle }}$ & $B_{\text {large }}$ \\
\hline$E_{\min }=100 E_{\mathrm{D}}$ & $5.7 \times 10^{3}$ & 0 & 40 & $6.8 \times 10^{2}$ & 0 & 0 & $2.0 \times 10^{3}$ & 0 & 0 \\
$E_{\min }=1000 E_{\mathrm{D}}$ & $9.1 \times 10^{4}$ & 0 & $2.4 \times 10^{4}$ & $5.4 \times 10^{4}$ & 0 & $9.8 \times 10^{3}$ & $4.7 \times 10^{4}$ & 0 & $1.1 \times 10^{4}$ \\
\hline$N_{\max }=1000$ & $B_{\text {small }}$ & $B_{\text {middle }}$ & $B_{\text {large }}$ & $B_{\text {small }}$ & $B_{\text {middle }}$ & $B_{\text {large }}$ & $B_{\text {small }}$ & $B_{\text {middle }}$ & $B_{\text {large }}$ \\
\hline$E_{\min }=100 E_{\mathrm{D}}$ & $5.3 \times 10^{4}$ & 0 & $1.7 \times 10^{3}$ & $1.4 \times 10^{4}$ & 0 & 0 & $2.2 \times 10^{4}$ & 0 & $2.8 \times 10^{2}$ \\
$E_{\min }=1000 E_{\mathrm{D}}$ & $3.9 \times 10^{5}$ & 0 & $1.5 \times 10^{5}$ & $2.9 \times 10^{5}$ & 0 & $8.4 \times 10^{4}$ & $2.2 \times 10^{5}$ & 0 & $7.9 \times 10^{4}$ \\
\hline
\end{tabular}

Table 6. Gamma ray lines fluences produced by inverse reactions of energetic $\mathrm{Ne}, \mathrm{Mg}, \mathrm{C}$, and $\mathrm{O}$ ions on ambient ${ }^{4} \mathrm{He}$.

\begin{tabular}{c|ccc|ccc}
\hline \hline & \multicolumn{3}{|c|}{$1.3639 \mathrm{MeV}$} & \multicolumn{3}{c}{$1.634 \mathrm{MeV}$} \\
\hline$N_{\max }=100$ & $B_{\text {small }}$ & $B_{\text {middle }}$ & $B_{\text {large }}$ & $B_{\text {small }}$ & $B_{\text {middle }}$ & $B_{\text {large }}$ \\
\hline$E_{\min }=100 E_{\mathrm{D}}$ & $2.0 \times 10^{3}$ & 0 & $2.3 \times 10^{2}$ & $3.1 \times 10^{3}$ & 0 & $2.8 \times 10^{2}$ \\
$E_{\min }=1000 E_{\mathrm{D}}$ & $8.7 \times 10^{3}$ & 0 & $4.2 \times 10^{3}$ & $1.8 \times 10^{4}$ & 0 & $7.9 \times 10^{3}$ \\
\hline$N_{\max }=1000$ & $B_{\text {small }}$ & $B_{\text {middle }}$ & $B_{\text {large }}$ & $B_{\text {small }}$ & $B_{\text {middle }}$ & $B_{\text {large }}$ \\
\hline$E_{\min }=100 E_{\mathrm{D}}$ & $1.0 \times 10^{4}$ & 0 & $2.5 \times 10^{3}$ & $2.0 \times 10^{4}$ & 0 & $3.0 \times 10^{3}$ \\
$E_{\min }=1000 E_{\mathrm{D}}$ & $1.8 \times 10^{4}$ & $1.2 \times 10^{2}$ & $1.4 \times 10^{4}$ & $4.7 \times 10^{4}$ & $1.4 \times 10^{2}$ & $3.2 \times 10^{4}$ \\
\hline
\end{tabular}

\begin{tabular}{c|ccc|ccc|ccc}
\hline \hline & \multicolumn{3}{|c|}{$4.438 \mathrm{MeV}(\mathrm{C})$} & \multicolumn{3}{c|}{$4.438 \mathrm{MeV}(\mathrm{O})$} & \multicolumn{3}{c}{$6.129 \mathrm{MeV}$} \\
\hline$N_{\max }=100$ & $B_{\text {small }}$ & $B_{\text {middle }}$ & $B_{\text {large }}$ & $B_{\text {small }}$ & $B_{\text {middle }}$ & $B_{\text {large }}$ & $B_{\text {small }}$ & $B_{\text {middle }}$ & $B_{\text {large }}$ \\
\hline$E_{\min }=100 E_{\mathrm{D}}$ & $3.4 \times 10^{3}$ & 0 & $1.3 \times 10^{2}$ & $2.3 \times 10^{3}$ & 0 & 31 & $7.2 \times 10^{2}$ & 0 & 0 \\
$E_{\min }=1000 E_{\mathrm{D}}$ & $2.7 \times 10^{4}$ & 0 & $1.0 \times 10^{4}$ & $1.8 \times 10^{4}$ & 0 & $7.0 \times 10^{3}$ & $5.5 \times 10^{3}$ & 0 & $2.1 \times 10^{3}$ \\
\hline$N_{\max }=1000$ & $B_{\text {small }}$ & $B_{\text {middle }}$ & $B_{\text {large }}$ & $B_{\text {small }}$ & $B_{\text {middle }}$ & $B_{\text {large }}$ & $B_{\text {small }}$ & $B_{\text {middle }}$ & $B_{\text {large }}$ \\
\hline$E_{\min }=100 E_{\mathrm{D}}$ & $2.4 \times 10^{4}$ & 0 & $2.4 \times 10^{3}$ & $1.8 \times 10^{4}$ & 0 & $9.5 \times 10^{2}$ & $5.5 \times 10^{3}$ & 0 & $2.9 \times 10^{2}$ \\
$E_{\min }=1000 E_{\mathrm{D}}$ & $7.9 \times 10^{4}$ & 0 & $4.7 \times 10^{4}$ & $5.1 \times 10^{4}$ & 0 & $3.3 \times 10^{4}$ & $1.5 \times 10^{4}$ & 0 & $9.9 \times 10^{3}$ \\
\hline
\end{tabular}

to the Dreicer field. These different values for the electric field normalization can be linked to the value of the magnetic field in the energy release region. The normalization to the Dreicer field considered in the simulation leads to minimum values of the magnetic field in the energy release region of $3 \mathrm{G}, 10 \mathrm{G}$, and $30 \mathrm{G}$ respectively. The maximum values that are rarely obtained are around 100 times these values. Extreme values of the magnetic fields are in this model at the origin of the acceleration of the highest energy particles that are not always efficiently produced in flares. The hard X-ray and gamma ray line fluxes produced by the energetic particle distributions in a thick target medium are finally computed and the results are compared with some observations from SMM, GRANAT, and RHESSI.

The main results of these simulations can be described as follows:

1. The final energy gain for electrons, protons, and heavier ions obviously depends on the energy gain in each reconnecting current sheet and thus on the assumption made for the longitudinal magnetic fields in the RCS. Thus, depending on these configurations, the present simulations can either reproduce electron dominated events or gamma-ray line events (see Fig. 8, but also the discussions in Litvinenko 1996).
Table 7. Relative abundances of $\mathrm{C}, \mathrm{Ne}, \mathrm{Mg}$, and $\mathrm{Si}$ to $\mathrm{O}$ (from Murphy et al. 1991).

\begin{tabular}{c|cccc}
\hline \hline Abundance & $\mathrm{C} / \mathrm{O}$ & $\mathrm{Ne} / \mathrm{O}$ & $\mathrm{Mg} / \mathrm{O}$ & $\mathrm{Si} / \mathrm{O}$ \\
\hline Photospheric & 0.43 & 0.14 & 0.044 & 0.041 \\
\hline Coronal & 0.41 & 0.136 & 0.19 & 0.177 \\
\hline
\end{tabular}

2. The energy contained in electrons above $20 \mathrm{keV}$ and protons above $1 \mathrm{MeV}$ for a total volume of the energy release/acceleration site containing distributed RCS of $100 \operatorname{arcsec}^{3}$ is consistent with what is deduced from the observations of HXR/GR flares (see the results of Fig. 8 for a volume of $1 \operatorname{arcsec}^{3}$ and observed values summarized in, e.g., Ramaty et al. 1995; and Mandzhavidze \& Ramaty 1996). The large efficiency for the acceleration process is linked to the assumption made of super Dreicer electric fields for every RCS that brings out a large fraction of the initial Maxwellian distribution to the non thermal energy range. The large dispersion of the relative electron and ion energy contents observed from one flare to the other (see, e.g., Vilmer \& MacKinnon 2003, for a review) can be also reproduced. Furthermore, in these simulations the existence of multiple acceleration regions solves the problem of the 


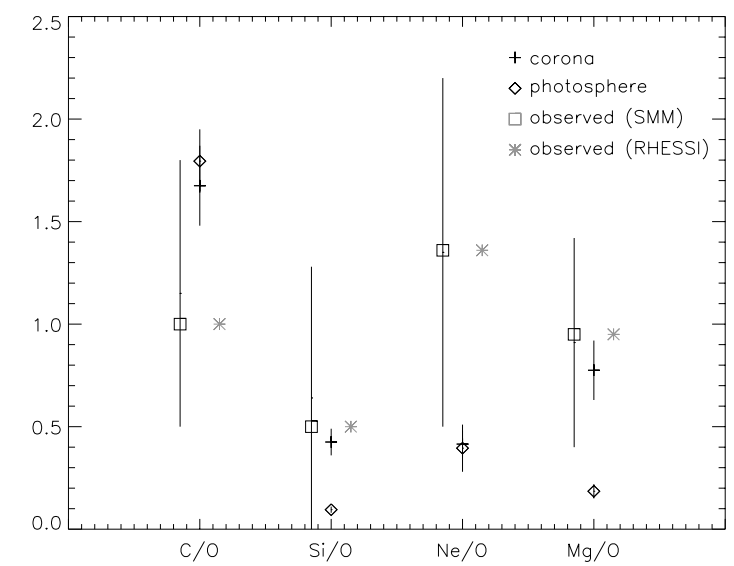

Fig. 10. Ratio of the direct gamma ray lines calculated in a thick target approximation for the proton and alpha energy distributions presented in Figs. 6 and 7. The diamond and cross marks represent the average calculated gamma ray line ratio for, respectively, photospheric and coronal ambient abundances. The vertical bars around these values show the range of the gamma-ray line ratio that can be obtained from the model for different electric field normalizations and magnetic field configurations. The square marks are deduced from the SMM observations of 19 flares (Share \& Murphy 1995). The vertical bars around these values represent the dispersion of the gamma ray ratio for the different flares. The stars represent the gamma ray ratio deduced from RHESSI observations during the 23 Jully 2002 event (Smith et al. 2003).

return current necessary to balance the current contained in the large number of energetic particles since particles are accelerated in distributed regions of the total acceleration volume and in different directions.

3. The final particle energy distribution has a shape similar to energy distributions found in other models based on multiple acceleration sites. Electron spectra presented in Anastasiadis et al. (2004) correspond here to electron acceleration in the case $B_{\text {large }}$. The shape of the case $B_{\text {total }}$ can be compared to the shape of the particle energy distribution found in the work of Turkmani et al. (2005). In this approach, particles are accelerated in a whole coronal loop by a highly fragmented electric field generated by a MHD code. In this last model, it can be considered that all kinds of magnetic configuration for the RCS can accelerate particles and results can be compared to the $B_{\text {total }}$ configuration. MHD codes cannot, however, deal with the particle acceleration processes occuring at small scales in each RCS and thus cannot predict differences between electron, proton, and heavy ion energy distributions. Flat particle spectra are obtained in the energy range around a few $\mathrm{keV}$ to several hundreds of $\mathrm{keV}$ followed by a strong softening of the spectra at higher energy. This is characteristic of any model invoking multiple particle acceleration sites that leads to an inverse exponential function since formally the particle trajectory is equivalent to a Levy walk.

4. The final particle energy distribution depends on the normalization of the electric field distribution with respect to the Dreicer electric field, on the number of interactions, and on the particle energy gain in each interaction site (i.e., on the assumed configuration for the RCS). Increasing the interaction number leads to an increase of the maximum final particle energy as well as to a flatter spectrum at low energies. These effects are similar for electrons, protons, and heavy ions. The main consequence on the particle energy distribution is the departure of the final particle distribution from the initial power law given by the energy release process. The spectral index of the final particle energy distribution will thus depend on the considered energy range. Figures $6 \mathrm{c}$ and $\mathrm{f}$ show for example that the proton spectral index in the energy ranges producing gamma-ray lines or, at much higher energies pion decay radiation, may be quite different, being steeper in the high energy domain. This is consistent with observational results from SMM (Murphy et al. 1987), GAMMA-1 and CGRO/COMPTEL (Kocharov et al. 1998), and PHEBUS/GRANAT (Vilmer et al. 2003), which all point to a steepening of the ion spectrum from the gamma-ray line to the pion-decay radiation producing energy ranges.

5. In some cases, the HXR flux produced by the simulated electron energy distributions is not realistic, being too flat at low energies and even exhibiting spectra increasing with energy. This results from the very flat shape of electron spectra, which in fact partly mimic the flat energy release distribution (see Fig. 3). Some of the simulations (mostly produced in the case of the minimum value of the normalization of the electric field distribution with respect to the Dreicer field (Figs. 9a and b)) may result in X-ray spectra similar to the ones observed in a few flares and with realistic energetic electron numbers if a total volume of $100 \operatorname{arcsec}^{3}$ is assumed for the energy release site. It must be noted that these spectra are consistent with the X-ray spectra obtained in Anastasiadis et al. (2004), and similar conclusions regarding the comparison between the results of the simulations and the observations can be drawn. Even in the best cases (Figs. 9a and b), the X-ray spectrum in the low energy part of the spectrum is flatter than observed on a statistical basis for HXR flares (e.g., Crosby et al. 1993; Bromund et al. 1995; Vestrand 1988). This may be due to the neglect of Coulomb energy losses in the model as well as to the flat spectrum of the energy release distribution. On the other hand, the values of the spectral indices in the photon energy range $100 \mathrm{keV}-1000 \mathrm{keV}$ appear reasonable when compared to observations (i.e., around $-2 ;-3$ ) in the same energy range from SMM (Vestrand 1988) and PHEBUS/GRANAT (e.g., Trottet et al. 1998; Vilmer et al. 1999). It must, however, be noticed that RHESSI observed a few photon spectra with spectral indices around $-2.2 \sim-2.3$ (still steeper than the simulations) in the $10-100 \mathrm{keV}$ range with a steepening towards -3 above $30 \mathrm{keV}$ (Krucker \& Lin 2002). Finally, a particular event observed by RHESSI (Kasparova et al. 2005) exhibited an unusually flat spectrum $(-1.5)$ in the $10-40 \mathrm{keV}$ range, steepening at higher energies, similar to what is found in some of the present simulations.

6. The gamma ray line fluences depend on the different normalizations of the electric field distributions, the number of interactions, and the RCS configurations. Observations of gamma-ray line ratios are mostly reproduced by our simulations for coronal target abundances, except for the neon to oxygen ratio, which is underestimated. However, it is well known that the neon line strength is uncertain because of the neon target abundance, which may be highly variable with respect to standard abundances.

As summarized above, the results of the present simulations can reproduce several observed properties of electrons and ions accelerated in solar flares and of their emissions: variable electron and ion energy content and $\gamma$-ray line ratio. However, the $\mathrm{X}$-ray spectra predicted in these simulations are usually too flat and result partly from the flatness of the energy release distribution. This can be intrinsic to models with many acceleration 
sites and with particles propagating freely between these acceleration sites and can be also attributed to several limitations of our model. First, we neglect the effect of the transport between the acceleration and the emission region. Secondly, we consider a very simple magnetic configuration of the RCS. We also have not considered the variation of energy gains in a RCS linked to the variation of the particle injection point, and we have thus made the simple assumption that the distribution of the acceleration lengths that will also determine the energy distribution of particles leaving the RCS is included in the random number $\alpha$. However, several works have shown that the acceleration of particles in RCS leads to power-law energy spectra when the dependence of the energy gain on the injection point is taken into account (Zharkova \& Gordovskyy 2005; Wood \& Neukirch 2005; Dalla \& Browning 2006). The next step in our model will then be to better describe the distribution of the particle acceleration lengths coming from the distribution of energy gains (Dauphin 2007). This would lead to different particle energy distributions and the flatness of the X-ray spectrum could be reduced by improving the estimations of the energy gains in the RCS.

Acknowledgements. The authors would like to thank L. Vlahos and M. Georgoulis for their helpful comments. In addition, A.A. would like to thank H. Isliker for useful discussions.

\section{References}

Anastasiadis, A. 2002, J. Atmosph. Solar-Terrestrial Phys., 64, 481 Anastasiadis, A., \& Vlahos, L. 1991, A\&A, 245, 271

Anastasiadis, A., \& Vlahos, L. 1994, ApJ, 428, 819

Anastasiadis, A., Vlahos, L., \& Georgoulis, M. 1997, ApJ, 489, 367

Anastasiadis, A., Gontikakis, C., Vilmer, N., \& Vlahos, L. 2004, A\&A, 422, 323

Bromund, K. R., McTiernan, J. M., \& Kane, S. R. 1995, ApJ, 455, 733

Brown, J. C. 1971, Sol. Phys., 18, 489

Crosby, N., Aschwanden, M., \& Dennis, B. 1993, Sol. Phys., 143, 275

Dalla, S., \& Browning, P. 2006, ApJ, 640, L99

Dauphin, C. 2007, A\&A, submitted

Decamp, N., \& Malara, F. 2006, ApJ, 637, L61

Dmitruk, P., Matthaeus, W., Seenu, N., \& Brown, M. 2003, ApJ, 597, 81

Dmitruk, P., Matthaeus, W., \& Seenu, N. 2004, ApJ, 617, 667

Efthimipoulos, C., Gontikakis, C., \& Anastasiadis, A. 2005, A\&A, 443, 663
Espagnet, O., Muller, R., Roudier, T., et al. 1993, A\&A, 271, 589 Fragos, T., Rantsiou, E., \& Vlahos, L. 2004, A\&A, 420, 719 Galsgaard, K., \& Nordlund, A. 1996, JGR, 101, A6, 13445

Georgoulis, M., \& Vlahos, L. 1998, A\&A, 336, 721

Gontikakis, C., Eftymiopoulos, C., \& Anastasiadis, A. 2006, MNRAS, 368, 293 Hughes, D., Psczuski, M., Dendy, R. O., et al. 2003, PhRvL, 90, 13, 131101 Isliker, H., Anastasiadis, A., Vassiliadis, D., et al. 1998, A\&A, 335, 1085 Isliker, H., Anastasiadis, A., \& Vlahos, L. 2001, A\&A, 377, 1068

Jackson, J. D. 1962, Classical Electrodynamics (New York: Wiley)

Kasparova, J., Karlicky, M., Kontar, E. P., et al. 2005, Sol. Phys., 232, 63

Kocharov, L., Debrunner, H., Kovaltsov, G., et al. 1998, A\&A, 340, 257

Krucker, S., \& Lin, R. P. 2002, Sol. Phys., 210, 229

Litvinenko, Y. E. 1996, ApJ, 462, 997

Litvinenko, Y. E. 2000, Sol. Phys., 194, 327

Litvinenko, Y. E., \& Somov, B. V. 1993, Sol. Phys., 146, 127

Mandzhavidze, N., \& Ramaty, R. 1996, Amer. Astron. Soc. Meet., 28, 858

Martens, P. C. H. 1988, ApJ, 330, 131

Martens, P. C. H., \& Young, A. 1990, ApJS, 73, 333

Miller, J. A., Cargill, P. J., Gordon, A. G., \& Dennis, B. R. 1997, JGR, 102, A7, 14631

Murphy, R. J., Dermer, C. D., \& Ramaty, R. 1987, ApJS, 63, 721

Murphy, R. J., Ramaty, R., Reames, D. V., et al. 1991, ApJ, 371, 793

Onofri, M., Isliker, H., \& Vlahos, L. 2006, PhRvL, 96, 1102

Parker, E. N. 1988, ApJ, 330, 474

Ramaty, R., Mandzhavidze, N., Kozlovsky, B., et al. 1995, ApJ, 455, 193

Saint-Hilaire, P., \& Benz, A. O. 2005, A\&A, 435, 743

Share, G. H., \& Murphy, R. J. 1995, ApJ, 452, 933

Smith, D. M., Share, G. H., Murphy, R. J., et al. 2003, ApJ, 595, 81

Speiser, T. W. 1965, JGR, 70, 4219

Trottet, G., Vilmer, N., Barat, C., et al. 1988, A\&A, 334, 1099

Trubnikov, B. A. 1965, Rev. Plasma Phys., 1, 105

Turkmani, R., Vlahos, L., Galsgaard, K., et al. 2005, ApJ, 620, L59

Vilmer, N., \& MacKinnon, A. L. 2003, in Energy Conversion and Particle Acceleration in the Solar Corona, ed. K. L. Klein (Heidleberg, Germany: Springer Verlag), 127

Vilmer, N., Trottet, G., Barrat, C., et al. 1999, A\&A, 342, 575

Vilmer, N., MacKinnon, A. L., Trottet, G., et al. 2003, A\&A, 412, 865

Vestrand, W. T. 1988, Sol. Phys., 118, 95

Vlahos, L., Georgoulis, M., Kluiving, R., \& Paschos, P. 1995, A\&A, 299, 897

Vlahos, L., Isliker, H., \& Lepreti, F. 2004, ApJ, 608, 540

Wood, P., \& Neukirch, T. 2005, Sol. Phys., 226, 73

Zharkova, V. V., \& Gordovskyy, M. 2004, ApJ, 604, 884

Zharkova, V. V., \& Gordovskyy, M. 2005a, MNRAS, 356, 3, 1107

Zharkova, V. V., \& Gordovsky, M. 2005b, Space Sci. Rev., 121, 165

Zhu, Z., \& Parks, G. 1993, JGR, 98, A5, 7603 